\title{
Dynamics of Entire Functions
}

\author{
Dierk Schleicher
}

\begin{abstract}
Complex dynamics of iterated entire holomorphic functions is an active and exciting area of research. This manuscript collects known background in this field and describes several of the most active research areas within the dynamics of entire functions.
\end{abstract}

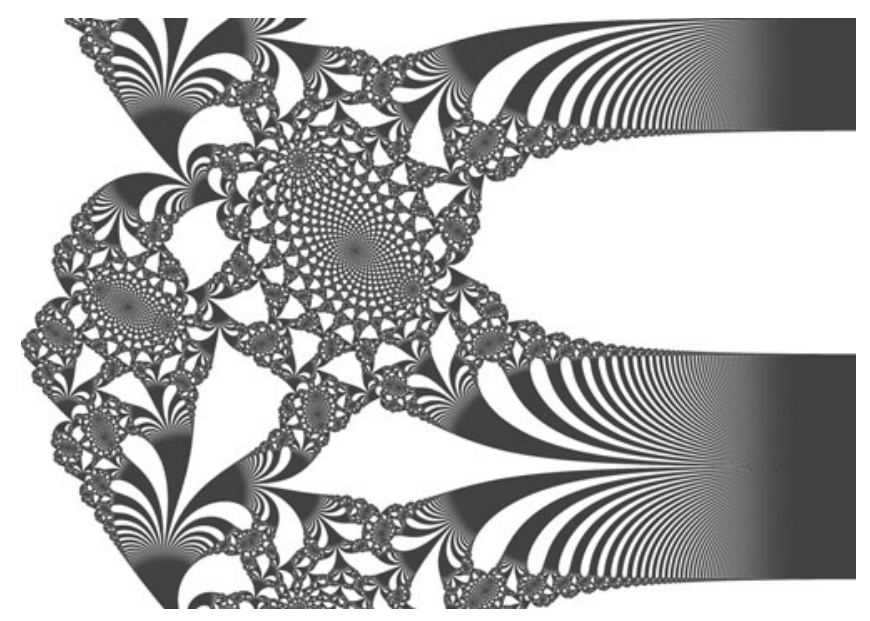

Complex dynamics, in the sense of holomorphic iteration theory, has been a most active research area for the last three decades. A number of interesting developments have taken place during this time. After the foundational work by Fatou and Julia in the early 20th century, which developed much of the basic theory of iterated general rational (and also transcendental maps), the advent of computer graphics

\section{Schleicher}

School of Engineering and Science, Research I, Jacobs University,

Postfach 750 561, D-28725 Bremen, Germany

e-mail: dierk@jacobs-university.de 
made possible detailed studies of particular maps; most often, quadratic polynomials as the simplest non-trivial holomorphic mappings were studied. While a number of deep questions on quadratic polynomials remain, interest expanded to specific (usually complex one- or two- dimensional) families of holomorphic maps, such as quadratic rational maps, cubic polynomials, or other families in which critical orbit relations reduced the space to simple families of maps: for instance, families of polynomials of degrees $d \geq 2$ with a single critical point of higher multiplicity. Only in recent years has the progress achieved so far allowed people to shift interest towards higher-dimensional families of iterated maps, such as general polynomials of degree $d \geq 2$. The study of iterated rational maps, as opposed to polynomials, seems much more difficult, mainly because of lack of a good partition to obtain a good encoding for symbolic dynamics: the superattracting fixed point at $\infty$, and the dynamic rays emanating from it, are important ingredients for deep studies of polynomials that are not available for general rational maps. A notable exception are rational maps that arise from Newton maps of polynomials: for these, it seems that good partitions for symbolic dynamics are indeed possible.

Transcendental iteration theory has been much less visible for a long time, even though its study goes back to Fatou (we will even treat a question of Euler in Section 4), and a solid body of knowledge has been developed by Baker and coauthors, and later also by Eremenko and Lyubich and by Devaney and coauthors, for more than four decades. Complex dynamics is known for employing methods from many different fields of mathematics, including geometry, complex analysis, algebra and even number theory. Transcendental dynamics unites two different general directions of research: there is a substantial body of knowledge coming from value distribution theory that often yields very general results on large classes of iterated transcendental functions; among the key contributors to this direction of research are Baker, Bergweiler, Eremenko, Rippon, and Stallard. The other direction of research sees transcendental functions as limits of rational functions and employs methods adapted from polynomial or rational iteration; here one usually obtains results on more specific maps or families of maps, most often the prototypical families of exponential or cosine maps; this direction of research was initiated by Devaney and coauthors. Others, like Lyubich and Rempe, have worked from both points of view.

In recent years, transcendental iteration theory has substantially gained interest. There have recently been international conferences specifically on transcendental iteration theory, and at more general conferences transcendental dynamics is obtaining more visibility.

In this survey article, we try to introduce the reader several aspects of transcendental dynamics. It is based on lecture notes of the CIME summer school held in Cosenza/Italy in summer 2008, but substantially expanded. The topic of that course, "dynamics of entire functions", also became the title of this article. We thus focus almost entirely on entire functions: their dynamical theory is much simpler than the theory of general meromorphic transcendental functions, much in the same way that polynomial iteration theory is much simpler (and more successful) than rational iteration theory. However, we believe that some of the successes of the polynomial 
theory still await to be carried over to the world of entire functions, and that some of the key tools (such as dynamic rays) are currently being developed.

In this survey article, we try to relate the two points of view on entire functions: that on large classes of entire functions and that on specific prototypical families of entire maps: the simplest families of maps are the exponential family $z \mapsto \lambda e^{z}$ with a single asymptotic value, and the cosine family $z \mapsto a e^{z}+b e^{-z}$ with two critical values. We try to cover several of the key topics in the theory of entire dynamics. This article is written for readers with a solid background in one-dimensional complex analysis, and a nodding acquaintance of complex dynamics of polynomials. Much more than what we need is provided in Milnor's now-classical book [Mi06].

In Section 1, we introduce the basic concepts of complex dynamics, including the Fatou and Julia sets, and more specifically the basic concepts of transcendental dynamics such as singular and asymptotic values. We introduce the important set $I(f)$ of escaping points, review some basic local fixed point theory, and describe the important Zalcman lemma with applications.

Section 2 discusses the possibilities for the Fatou set of entire functions and especially highlight those features that are not known from the rational or polynomial theory: Baker domains, wandering domains, and "Baker wandering domains".

The space of general entire functions is a huge space, and many results require the restriction to smaller families of maps: sometimes because tools are lacking to prove results in greater generality, but sometimes also because the space of entire functions is so big that many different dynamical properties are possible, and satisfactorily strong results are possible only when restricting to maps with specific properties. Section 3 introduces important classes of entire maps that are often useful, especially the Eremenko-Lyubich class $B$ of entire functions of bounded type and the class $S$ of functions of finite type.

Section 4 is an overview on results on the set $I(f)$ of escaping points: just as for polynomials, these points often have useful properties that are comparatively easy to investigate, and thus make it possible to establish interesting properties of the Julia set (and sometimes the Fatou set).

Section 5 discusses a number of properties on the Hausdorff dimension of Julia set; this is a rich and active area with a number of interesting and sometimes surprising results.

Section 6 is a brief introduction to parameter spaces of entire functions. We briefly describe a general result on natural parameter spaces of entire functions, and then discuss exponential parameter space as the best-studied parameter space and prototypical parameter space of entire functions, in analogy to the Mandelbrot set for quadratic polynomials.

Section 7 is not directly concerned with the dynamics of entire functions, but with Newton methods of entire functions: these are special meromorphic functions; we hope that, just as in the rational case, these will be meromorphic functions that can be investigated relatively easily; we describe a number of known results on them.

In Section 8, we list a small number of questions that remain open in the field: some of them have remained open for a long time, while others are new. A research area remains lively as long as it still has open questions. 
In a brief appendix, we state a few important theorems from complex analysis that we use throughout.

The research field of complex dynamics is large and active, and many people are working on it from many different points of view. We have selected some topics that we find particularly interesting, and acknowledge that there are a number of other active and interesting topics that deserve no less attention. We mention in particular results on measure theory, including the thermodynamic formalism (see Urbański [Ur03] for a recent survey). Further important omitted areas that we should mention are Siegel disks and their boundaries (see e.g., Rempe [Re04]), questions of linearizations and small cycles (see e.g. Geyer [Ge01]), the construction of entire maps with specific geometric or dynamic properties (here various results of Bergweiler and Eremenko should be mentioned), the relation of transcendental dynamics to Nevanlinna theory, and Thurston theory for transcendental maps (see Selinger [Se09]).

We tried to give many references to the literature, but are acutely aware that the literature is vast, and we apologize to those whose work we failed to mention. Many further references can be found in the 1993 survey article of Bergweiler [Be93] on the dynamics of meromorphic functions.

The illustration on the first page shows the Julia set of a hyperbolic exponential map with an attracting periodic point of period 26. The Fatou set is in white. We thank Lasse Rempe for having provided this picture.

ACKNOWLEDGEMENTS. I would like to thank my friends and colleagues for many interesting and helpful discussions we have had on the field, and in particular on drafts of this manuscript. I would like to especially mention Walter Bergweiler,Jan Cannizzo, Yauhen Mikulich, Lasse Rempe, Phil Rippon, Nikita Selinger, and Gwyneth Stallard. And of course I would like to thank the CIME foundation for having made possible the summer school in Cosenza, and Graziano Gentili and Giorgio Patrizio for having made this such a memorable event!

\section{Fatou and Julia Set of Entire Functions}

Throughout this text, $f$ will always denote a transcendental entire function $f: \mathbb{C} \rightarrow \mathbb{C}$. The dynamics is to a large extent determined by the singular values, so we start with their definition.

\section{Definition 1.1 (Singular Value).}

A critical value is a point $w=f(z)$ with $f^{\prime}(z)=0$; the point $z$ is a critical point. An asymptotic value is a point $w \in \mathbb{C}$ such that there exists a curve $\gamma:[0, \infty) \rightarrow \mathbb{C}$ so that $\gamma(t) \rightarrow \infty$ and $f(\gamma(t)) \rightarrow w$ as $t \rightarrow \infty$. The set of singular values of $f$ is the closed set

$$
S(f):=\overline{\{\text { critical and asymptotic values }\}} .
$$

This definition is not completely standard: some authors do not take the closure in this definition. 
A postsingular point is a point on the orbit of a singular value.

In any dynamical system, it is useful to decompose the phase space (in this case, the dynamical plane $\mathbb{C}$ ) into invariant subsets. In our case, we will mainly consider the Julia set $J(f)$, the Fatou set $F(f)$, and the escaping set $I(f)$, as well as certain subsets thereof.

\section{Definition 1.2 (Fatou and Julia Sets).}

The Fatou set $F(f)$ is the set of all $z \in \mathbb{C}$ that have a neighborhood $U$ on which the family of iterates $f^{\circ n}$ forms a normal family (in the sense of Montel; see Definition A.1). The Julia set $J(f)$ is the complement of the Fatou set: $J(f):=\mathbb{C} \backslash F(f)$. A connected component of the Fatou set is called a Fatou component.

\section{Definition 1.3 (The Escaping Set).}

The set $I(f)$ is the set of points $z \in \mathbb{C}$ with $f^{\circ n}(z) \rightarrow \infty$.

Clearly, $F(f)$ is open and $J(f)$ is closed, while in general, $I(f)$ is neither open nor closed. All three sets $J(f), F(f)$, and $I(f)$ are forward invariant, i.e., $f(F(f)) \subset$ $F(f)$ etc.; this is true by definition. Note that equality may fail when $f$ has omitted values: for instance for $z \mapsto 0.1 e^{z}$, the Fatou set contains a neighborhood of the origin, but 0 is an omitted value. It is easy to see that for each $n \geq 1, F\left(f^{\circ n}\right)=F(f)$ and hence $J\left(f^{\circ n}\right)=J(f)$, and of course $I\left(f^{\circ n}\right)=I(f)$.

\section{Theorem 1.4 (The Julia Set).}

The Julia set is non-empty and unbounded and has no isolated points.

The Fatou set may or may not be empty. An example of an entire function with empty Fatou set is $z \mapsto e^{z}$ [Mis81]. More generally, the Fatou set is empty for every entire function of finite type (see Definition 3.1) for which all singular values are either preperiodic or escape to $\infty$ (Corollary 3.14). We will describe the Fatou set, and give examples of different types of Fatou components, in Section 2. The Fatou set is non-empty for instance for any entire function with an attracting periodic point.

The fact that $J(f)$ is non-empty was established by Fatou in 1926 [Fa26]. We give here a simple proof due to Bargmann [Ba99]. We start with a preparatory lemma.

\section{Lemma 1.5 (Existence of Periodic Points).}

Every entire function, other than a polynomial of degree 0 or 1 , has at least two periodic points of period 1 or 2 .

Remark 1.6. This is a rather weak result with a simple proof; a much stronger result is given, without proof, in Theorem 1.21.

Proof. Consider an entire function $f$ and define a meromorphic function via $g(z):=$ $(f \circ f(z)-z) /(f(z)-z)$.

Suppose that $g$ is constant, say $g(z)=c$ for all $z \in \mathbb{C}$, hence $f \circ f(z)-z=$ $c(f(z)-z)$. If $c=0$, then $f \circ f=\mathrm{id}$, so $f$ is injective and thus a polynomial of degree 1 . If $c=1$, then $f \circ f=f$, so for each $z \in \mathbb{C}$, the value $f(z)$ is a fixed point of $f$ : this implies that either $f=$ id and every $z \in \mathbb{C}$ is a fixed point, or fixed points 
of $f$ are discrete and thus $f$ is constant. If $c \notin\{0,1\}$, then differentiation yields $\left(f^{\prime} \circ f\right) f^{\prime}-1=c f^{\prime}-c$ or $f^{\prime}\left(f^{\prime} \circ f-c\right)=1-c$. Since $c \neq 1$, it follows that $f^{\prime}$ omits the value 0 and $f^{\prime} \circ f$ omits the value $c \neq 0$, so $f^{\prime}$ can assume the value $c$ only at the omitted values of $f$. By Picard's Theorem A.4, it follows that $f^{\prime}$ is constant and thus $f$ is a polynomial of degree at most 1 .

In our case, $f$ is not a polynomial of degree 0 or 1 by hypothesis, so $g$ is a non-constant meromorphic function. Suppose $p \in \mathbb{C}$ is such that $g(p) \in\{0,1, \infty\}$. If $g(p)=\infty$, then $f(p)=p$; if $g(p)=0$, then $f(f(p))=p$; and if $g(p)=1$, then $f(f(p))=f(p)$.

If $g$ is transcendental, then by Picard's theorem there are infinitely many $p \in \mathbb{C}$ with $g(p) \in\{0,1, \infty\}$. If $g$ is a non-constant rational map (which in fact never happens), then there are $p_{0}, p_{1}, p_{\infty} \in \overline{\mathbb{C}}$ with $g\left(p_{i}\right)=i$, and at least two of them are in $\mathbb{C}$.

As an example, the map $f(z)=e^{z}+z$ has no fixed points; in this case $g(z)=$ $e^{e^{z}}+1$ has no $p \in \mathbb{C}$ with $g(p) \in\{1, \infty\}$, but of course infinitely many $p$ with $g(p)=0$ (corresponding to periodic points of period 2).

Proof of Theorem 1.4. Let $f$ be an entire function other than a polynomial of degree 0 or 1 . By Lemma $1.5, f$ has (at least) two periodic points of period 1 or 2 ; replacing $f$ by $f \circ f$ if necessary, we may suppose that $f$ has two fixed points, say at $p, p^{\prime} \in \mathbb{C}$ (note that $J(f)=J(f \circ f)$ ). If $\left|f^{\prime}(p)\right|>1$, then $p \in J(f)$. In order to show that $J(f) \neq \emptyset$, we may assume that $p \in F(f)$ and in particular that $\left|f^{\prime}(p)\right| \leq 1$. Let $W$ be the Fatou component containing $p$.

If $\left|f^{\prime}(p)\right|=1$, then any limit function of the family of iterates $\left\{\left.f^{\circ n}\right|_{W}\right\}$ is nonconstant. So let $f^{\circ n_{j}}$ be a subsequence that converges to a non-constant limit function; then $f^{\circ\left(n_{j+1}-n_{j}\right)}$ converges to the identity on $W$, and this implies that $f \mid W$ is injective. If $W=\mathbb{C}$, then this is a contradiction to the choice of $f$, hence $F(f) \neq \mathbb{C}$.

The final case we have to consider is $\left|f^{\prime}(p)\right|<1$; for convenience, we may suppose that $p=0$. If $F(f)=\mathbb{C}$, then $f^{\circ n} \rightarrow 0$ uniformly on compacts in $\mathbb{C}$. We will show that $f$ is a polynomial of degree at most 1 . Let $D$ be an open disk centered at $p$ such that $f(D) \subset D$. For $N \in \mathbb{N}$, let $D_{n}:=f^{\circ(-n)}(D)$; since $D$ is simply connected, it follows that also $D_{n}$ is simply connected. Let $r_{n}$ be maximal so that the round circle $\partial D_{r_{n}}(0) \subset \overline{D_{n}}$; this implies that $D_{r_{n}} \subset D_{n}$. We have $D_{n} \subset D_{n+1}$ and $\bigcup_{n} D_{n}=\mathbb{C}$, hence $r_{n+1} \geq r_{n}$ and $r_{n} \rightarrow \infty$.

As usual, for $r>0$, define $M(r ; f):=\max \{|f(z)|:|z|=r\}$. For each $n \in \mathbb{N}$, define maps $h_{n}: \mathbb{D} \rightarrow \mathbb{C}$ via $h_{n}(z)=M\left(r_{n} / 2 ; f\right)^{-1} f\left(r_{n} z\right)$. All $h_{n}$ satisfy $h_{n}(0)=0$ and $M\left(1 / 2 ; h_{n}\right)=1$. Let

$$
c_{n}:=\sup \left\{r>0: \partial D_{r}(0) \subset h_{n}(\mathbb{D})\right\} .
$$

If some $c_{n}$ satisfies $c_{n}<1$, then there are points $a_{n}, b_{n} \in \mathbb{C}$ with $\left|a_{n}\right|=1,\left|b_{n}\right|=2$ so that $h_{n}(\mathbb{D}) \cap\left\{a_{n}, b_{n}\right\}=\emptyset$. If this happens for infinitely many $n$, then we can extract a subsequence with $c_{n}<1$, and it follows easily from Montel's theorem that the $h_{n}$ form a normal family. After extracting another subsequence, we may suppose that the $h_{n}$ converge to a holomorphic limit function $h: \mathbb{D} \rightarrow \mathbb{C}$ that inherits from the $h_{n}$ the properties that $h(0)=0$ and $M(1 / 2 ; h)=1$. Thus $h$ is non-constant and there is 
an $r>0$ with $D_{r}(0) \subset h(\mathbb{D})$. But then $c_{n}>r / 2$ for all large $n$. This implies that no subsequence of the $c_{n}$ tends to 0 , and hence that $\inf \left\{c_{n}\right\}>0$.

There is thus a $c>0$, and there are $c_{n}^{\prime}>c$ with $\partial D_{c_{n}^{\prime}}(0) \subset h_{n}(\mathbb{D})$ for all large $n$. This implies

$$
\partial D_{c_{n}^{\prime} M\left(r_{n} / 2 ; f\right)}(0) \subset f\left(D_{r_{n}}(0)\right) \subset f\left(D_{n}\right) \subset D_{n}
$$

and thus, by the definition of $r_{n}$,

$$
c M\left(r_{n} / 2 ; f\right)<r_{n}
$$

Therefore, $M\left(r_{n} / 2 ; f\right) / r_{n}$ is bounded.

Define an entire function $g$ via $g(z):=f(z) / z-f^{\prime}(0)$ (the isolated singularity at $z=0$ is removable because $f(0)=0$, and thus $g(0)=0)$. It has the property that $M\left(r_{n} / 2 ; g\right)$ is bounded; since $r_{n} \rightarrow \infty$, this implies that $g$ is bounded on $\mathbb{C}$ and hence constant. But $g(0)=0$, hence $f(z)=z f^{\prime}(0)$ for all $z$, so $f$ is a polynomial of degree at most 1 as claimed.

We have now shown that, if $f$ is not a polynomial of degree 0 or 1 , then $J:=$ $J(f) \neq \emptyset$. If $|J|>1$, then it follows from Picard's Theorem A.4 that $J$ is infinite and unbounded: as soon as $J$ contains at least two points $a, a^{\prime}$, every neighborhood of $\infty$ contains infinitely many preimages of $a$ or $a^{\prime}$.

We finally have to consider the case that $|J|=1$, say $J=\{a\}$. In this case, $f(a)=$ $a$, and $f$ must have another fixed point, say $p$, and $W:=\mathbb{C} \backslash\{a\}$ equals the Fatou set. We must have $\left|f^{\prime}(p)\right| \leq 1$, and $\left|f^{\prime}(p)\right|=1$ would lead to the same contradiction as above. This implies that $\left|f^{\prime}(p)\right|<1$. But any loop in $W$ starting and ending at $p$ would converge uniformly to $p$, and by the maximum modulus principle this would imply that $W$ was simply connected, a contradiction. This shows that $|J|>1$ in all cases, hence that $J$ is always an infinite set (we will show below in Theorem 1.7 that $J$ is always uncountable).

For an entire function $f$, an exceptional point is a point $z \in \mathbb{C}$ with finite backwards orbit. There can be at most one exceptional point: in fact, any finite set of exceptional points must have cardinality at most 1 (or the complement to the union of their backwards orbits would be forward invariant and hence contained in the Fatou set, by Montel's Theorem A.2, so the Julia set would be finite). If an entire function $f$ has an exceptional point $p$, it is either an omitted value or a fixed point (such as the point 0 for $e^{z}$ or $z e^{z}$ ); in such cases, $f$ restricts to a holomorphic self-map of the infinite cylinder $\mathbb{C} \backslash\{p\}$.

\section{Theorem 1.7 (Topological Properties of the Julia Set).}

For every entire function $f$ (other than polynomials of degree 0 or 1), the Julia set is the smallest closed backward invariant set with at least 2 points. The Julia set is contained in the backwards orbit of any non-exceptional point in $\mathbb{C}$, it has no isolated points, and it is locally uncountable.

Proof. Suppose $z \in \mathbb{C}$ has a neighborhood $U$ so that $f^{\circ n}(U)$ avoids $a$. Then it also avoids all points in $f^{-1}(a)$. Unless $a$ is an exceptional point, Montel's Theorem A.2 implies that $z$ is in the Fatou set. The backwards orbit of any non-exceptional point in $\mathbb{C}$ thus accumulates at each point in the Julia set. Any closed backwards invariant 
set with at least two elements thus contains $J(f)$. The Julia set itself is closed and backward invariant and contains at least two points by Theorem 1.4, so it is the smallest such set.

Since any point in $J(f)$ is the limit point of backwards orbits of points in $J(f)$, it follows that no point in $J(f)$ is isolated. Any closed set without isolated points is locally uncountable.

\section{Theorem 1.8 (Julia Set and Escaping Set).}

For every entire function $f$ (other than polynomials of degree 0 or 1 ), the set $I(f)$ is infinite, and $J(f)=\partial I(f)$.

Sketch of proof. The hard part consists in showing the existence of an escaping point; this implies that $I(f)$ is infinite. Using Montel's Theorem A.2, this implies that the Fatou set contains every open subset of $\mathbb{C} \backslash I(f)$ (if any). We have $|\mathbb{C} \backslash I(f)| \geq 2$ (for instance, because there exist periodic points; see Lemma 1.5), so for the same reason, any open subset of $I(f)$ is also part of the Fatou set. This implies $J(f) \subset$ $\partial I(f)$. Conversely, if $z \in I(f) \cap F(f)$, then any limit function of the iterates near $z$ must have the value $\infty$; but locally uniform limits of entire functions are either entire or constant equal to $\infty$, and $z \in I(f) \backslash \partial I(f)$.

The fact that every transcendental entire function has escaping points was shown by Eremenko [Er89] using Wiman-Valiron-theory (a different proof, based on a theorem of Bohr, is due to Domínguez [Do98]). While the details are quite technical, the idea of Eremenko's proof can be described as follows (here we follow an exposition of Bergweiler). Write $f(z)=\sum_{n>0} a_{n} z^{n}$. For a radius $r>1$, define the central index $v=v(r, f)$ so that $\left|a_{v}\right| r^{v}=\max _{n>0}\left\{\left|a_{n}\right| r^{n}\right\}$. Choose $z_{r}$ so that $\left|f\left(z_{r}\right)\right|$ is maximal among all $z$ with $|z|=r$. Then Wiman and Valiron showed that $f(z) \asymp\left(z / z_{r}\right)^{v} f\left(z_{r}\right)$ for $z$ close to $z_{r}$, where "こ" means "the quotient is bounded" (more precisely, choosing some $\tau>1 / 2$, this holds if $\left|z-z_{r}\right|<r / v^{\tau}$, and only if $r$ is outside an exceptional set $F$ that is small enough so that $\left.\int_{F} d t / t<\infty\right)$. We will assume that $\tau \in(1 / 2,1)$.

We write $z=z_{r} e^{w}$ and obtain $f\left(z_{r} e^{w}\right) \asymp e^{w v} f\left(z_{r}\right)$ if $|w|<1 / v^{\tau}$ and $r \notin F$, in particular if $|\operatorname{Re} w|<\pi / v,|\operatorname{Im} w|<\pi / v$ (if $v$ is sufficiently large, then $\pi / v<$ $\left.1 / \boldsymbol{v}^{\boldsymbol{\tau}}\right)$. The corresponding square around $z_{r}$ maps to a large annulus around $f\left(z_{r}\right)$ at absolute value much greater than that of $z_{r}$. In the image, the argument can be repeated, and this yields an escaping orbit (in each step, we need to exclude values of $r$ from the exceptional set $F$, and estimate that enough values of $r$ remain).

This result has been generalized, by a different but related method, by Bergweiler, Rippon, Stallard [BRS08], to show the existence of points that escape through a specific sequence "tracts" of $f$.

\section{Definition 1.9 (Local Fixed Point Theory).}

Consider a local holomorphic function $g: U \rightarrow \mathbb{C}$ with $U \subset \mathbb{C}$ open, and a fixed point $p \in U$ with derivative $\mu:=g^{\prime}(p)$. Then $\mu$ is called the multiplier of the fixed point $p$, and $p$ is called

attracting if $|\mu|<1$ (and superattracting if $\mu=0$ );

repelling if $|\mu|>1$; 
indifferent if $|\boldsymbol{\mu}|=1$; in particular

rationally indifferent (or parabolic) if $\mu$ is a root of unity;

irrationally indifferent if $|\mu|$ is indifferent but not a root of unity.

\section{Theorem 1.10 (Local Fixed Point Theory).}

Local holomorphic maps have the following normal forms near fixed points:

- in a neighborhood of a superattracting fixed point, the map is conformally conjugate to $z \mapsto z^{d}$ near 0 , for a unique $d \geq 2$;

- in a neighborhood of an attracting or repelling fixed point with multiplier $\mu$, the map is conformally conjugate to the linear map $z \mapsto \mu z$ near 0 ("attracting and repelling fixed points are locally linearizable");

- local normal forms in a neighborhood of parabolic fixed points are complicated (see Abate [Ab]; but within each attracting petal, the map is conformally conjugate to $z \mapsto z+1$ in a right half plane;

- an irrationally indifferent fixed point p may or may not be linearizable.

Remark 1.11. This is a local result; for a proof, see [Mi06]. If an irrationally indifferent fixed point is linearizable, a maximal linearizable neighborhood is called a Siegel disk; this is a Fatou component. There is a sufficient condition, due to Yoccoz, that assures linearizability of a local holomorphic map (and in particular of an entire function) with an irrationally indifferent fixed point, depending only on the multiplier [Mi06]. Non-linearizable irrationally indifferent fixed points are called Cremer points; they are in the Julia set and not associated to any type of Fatou component. (As a result, if the Julia set equals $\mathbb{C}$, then all fixed points are repelling or Cremer.)

Remark 1.12. The same classification applies of course to periodic points: these are fixed points of appropriate iterates.

In Definition 1.1 we had defined a singular value to be a critical or asymptotic value, or a limit point thereof. Now we discuss singular values somewhat more closely, following Iversen [Iv14]; see also [BE95, Ne53]. Choose a point $a \in \overline{\mathbb{C}}$. For each $r>0$, let $U_{r}$ be a component of $f^{-1}\left(D_{\chi}(a, r)\right)$ (where $D_{\chi}(a, r)$ is the $r$-neighborhood of $a$ with respect to the spherical metric), chosen so that $U_{r^{\prime}} \subset U_{r}$ for $r^{\prime}<r$. Then either $\bigcap_{r} U_{r}=\{z\}$ for a unique $z \in \mathbb{C}$, or $\bigcap_{r} U_{r}=\emptyset$ (the set $\bigcap_{r} U_{r}$ is connected; if it contains more than one point, then $f$ is constant). This collection $\left\{U_{r}\right\}$ is called a tract for $f$. If $\bigcap_{r} U_{r}=\{z\}$, then $f(z)=a$, and $a$ is a regular value for this tract if $f^{\prime}(z) \neq 0$, and $a$ is a critical value if $f^{\prime}(z)=0$. If $\bigcap_{r} U_{r}=\emptyset$, then $a$ is an asymptotic value for this tract, and there exists a curve $\gamma:[0, \infty) \rightarrow \mathbb{C}$ with $\gamma(t) \rightarrow \infty$ through $U_{r}$ and $f(\gamma(t)) \rightarrow a$ (as in Definition 1.1); such a path $\gamma$ is called an asymptotic path. Of course, for the same function $f$, the same point $a \in \mathbb{C}$ can be a regular or singular value of different types for different tracts.

Critical values are called algebraic singularities, and asymptotic values are called transcendental singularities (for a particular choice of the tract). Any tract with $\bigcap U_{r}=\emptyset$ is called an asymptotic tract.

An asymptotic value is a direct singularity for a tract $\left\{U_{r}\right\}$ if there is an $r>0$ so that $f\left(U_{r}\right) \not \supset a$, and an indirect singularity otherwise. A direct singularity is a 
logarithmic singularity if there is an $U_{r}$ so that $f: U_{r} \rightarrow D_{\chi}(a, r) \backslash\{a\}$ is a universal covering. In particular, if $f$ is a transcendental entire function, then $\infty$ is always a direct asymptotic value (see Theorem 1.14 below). For entire functions of bounded type (see Section 3 ), $\infty$ is always a logarithmic singularity.

Recall from Definition 1.1 that we defined singular value to be a critical value, an asymptotic value, or a limit point thereof.

\section{Theorem 1.13 (Singular Values).}

Any $a \in \mathbb{C}$ that is not a singular value has a neighborhood $U$ so that $f: f^{-1}(U) \rightarrow U$ is an unbranched covering (i.e., a is a regular value for all tracts).

Proof. Choose a neighborhood $U$ of $a$, small enough so that it is disjoint from $S(f)$; it thus contains no critical or asymptotic value. If $U$ has an unbounded preimage component, then it is not hard to show that $U$ contains an asymptotic value (successively subdivide $U$ so as to obtain a nested sequence of open sets with diameters tending to 0 but with unbounded preimages). Therefore, $a$ has a simply connected neighborhood for which all preimage components are bounded. If $V$ is such a preimage component, then $f: V \rightarrow U$ is a branched covering, and if $U$ contains no critical value, then $f: V \rightarrow U$ is a conformal isomorphism. The claim follows.

The set of direct asymptotic values is always countable [He57] (but the number of associated asymptotic tracts need not be). There are entire functions for which every $a \in \mathbb{C}$ is an asymptotic value [Gr18b]. On the other hand, according to the Gross Star Theorem [Gr18a], every entire function $f$ has the property that for every $a \in \mathbb{C}$ and for every $b \in \mathbb{C}$ with $f(b)=a$, and for almost every direction, the ray at $a$ in this direction can be lifted under $f$ to a curve starting at $b$.

The following theorem of Iversen is important.

\section{Theorem 1.14 (Omitted Values are Asymptotic Values).}

If some $a \in \overline{\mathbb{C}}$ is assumed only finitely often by some transcendental entire function $f$, then a is a direct asymptotic value of $f$. In particular, for every entire function, $\infty$ is always a direct asymptotic value.

Proof. For any $r>0$, any bounded component of $f^{-1}\left(D_{\chi}(r, a)\right)$ contains a point $z$ with $f(z)=a$. Since by Picard's Theorem A.4, at most one point in $\mathbb{C}$ is assumed only finitely often, it follows that for every $r>0$, the set $f^{-1}\left(D_{\chi}(r, a)\right)$ cannot consist of bounded components only. Therefore, for each $r>0$, there is at least one unbounded component, and thus at least one asymptotic tract over the asymptotic value $a$; for such a tract, $a$ is a direct singularity. Since the point $\infty$ is omitted for each entire function, it is a direct asymptotic value.

The following definition is of great importance in function theory:

\section{Definition 1.15 (Order of Growth).}

The order of an entire function $f$ is

$$
\text { ord } f:=\limsup _{z \rightarrow \infty} \frac{\log \log |f|}{\log |z|} .
$$


(Sometimes, one also uses the lower order of $f$; for this definition, the limsup is replaced by the liminf.)

Remark 1.16. The prototypical example of a function of finite order $d \in \mathbb{N}$ is $z \mapsto$ $\exp \left(z^{d}\right)$. Unlike the properties of finite or bounded type, the property of finite order is not preserved under compositions, and was thus thought to be of limited use in dynamics; however, the order of growth has recently found important applications in dynamics, for instance when dynamical properties are controlled using geometric function theory (for instance, in Theorem 4.11 below).

The finite order condition also comes into play in the discussion of direct and indirect asymptotic values.

\section{Theorem 1.17 (Denjoy-Carleman-Ahlfors).}

For any function $f$ of finite order, the number of asymptotic values, and even the number of different asymptotic tracts, is at most $\max \{1,2 \operatorname{ord} f\}$ and hence finite. More precisely, if $f$ has $m$ direct and $n$ indirect asymptotic tracts, then $2 m+n \leq$ $\max \{1,2 \operatorname{ord} f\}$.

In fact, between any two different asymptotic tracts of finite asymptotic values, there must be an asymptotic tract of the asymptotic value $\infty$; and if the latter is very narrow, then $f$ must grow very quickly along this tract; the extra count for direct asymptotic tracts comes in because these, too, need to have certain width (postcomposing $f$ with a Möbius transform preserves the order, and can turn direct tracts over finite asymptotic values to tracts over $\infty$ ). Compare [Ne53, Sec. 269].

\section{Theorem 1.18 (Indirect Asymptotic Values and Critical Values).}

For any function of finite order, every indirect asymptotic value is a limit point of critical values.

For a proof, see [BE95].

The following result from [Z75] is often useful in complex dynamics, especially transcendental dynamics.

\section{Lemma 1.19 (The Zalcman Lemma).}

Let $U \subset \mathbb{C}$ be any domain and let $F$ be a non-normal family of holomorphic functions from $U$ to $\mathbb{C}$. Then there exist a non-constant entire function $g: \mathbb{C} \rightarrow \mathbb{C}$ and sequences $f_{k} \in F, z_{k} \in U$ and $\rho_{k}>0$ with $\rho_{k} \rightarrow 0$ so that $z_{k} \rightarrow z_{\infty} \in U$ and

$$
g_{k}(\zeta):=f_{k}\left(z_{k}+\rho_{k} \zeta\right) \longrightarrow g(\zeta)
$$

uniformly on compacts in $\mathbb{C}$. If $a \in U$ is such that the restriction of $F$ to any neighborhood of a is not normal, then we may require that $z_{n} \rightarrow a$.

Proof. After rescaling, we may suppose that $\overline{\mathbb{D}} \subset U$ and that the point $a=0$ has the property that the restriction of $F$ to any neighborhood of 0 is not normal. Let $\left(f_{k}\right) \subset F$ be any sequence that does not form a normal family when restricted to any neighborhood of $a$. 
According to Marty's criterion (Theorem A.3), a family of maps $\left(f_{k}\right)$ is normal if and only if its spherical derivatives $f^{\sharp}(z)=\left|f^{\prime}(z)\right| /\left(1+|f(z)|^{2}\right)$ are locally bounded. Since this is not the case, one can extract a subsequence of the $f_{k}$ and then a sequence $\zeta_{k} \rightarrow 0$ with $f_{k}^{\sharp}\left(\zeta_{k}\right) \rightarrow \infty$. Choose asequence $\left(r_{k}\right) \subset \mathbb{R}^{+}$so that $r_{k} \rightarrow 0$ but $r_{k} f_{k}^{\sharp}\left(\zeta_{k}\right) \rightarrow$ $\infty$; we may assume that $\left|\zeta_{k}\right|+2 r_{k}<1$ for all $k$.

Find a sequence $z_{k} \in \mathbb{C}$ with $\left|z_{k}-\zeta_{k}\right|<r_{k}$ so that

$$
M_{k}:=f_{k}^{\sharp}\left(z_{k}\right)\left(1-\left|z_{k}-\zeta_{k}\right| / r_{k}\right)=\sup _{\left|z-\zeta_{k}\right|<r_{k}} f_{k}^{\sharp}(z)\left(1-\left|z-\zeta_{k}\right| / r_{k}\right) .
$$

This implies $z_{k} \rightarrow 0$ and $f_{k}^{\sharp}\left(z_{k}\right) \geq M_{k} \geq f_{k}^{\sharp}\left(\zeta_{k}\right)$. Extract a subsequence so that $f_{k}\left(z_{k}\right)$ converges to a limit $b \in \overline{\mathbb{C}}$.

Define $\rho_{k}:=1 / f_{k}^{\sharp}\left(z_{k}\right)=\left(1-\left|z_{k}-\zeta_{k}\right| / r_{k}\right) / M_{k}$; then $\rho_{k} \rightarrow 0$. Define functions $g_{k}:\left\{z \in \mathbb{C}:|z|<r_{k} / \rho_{k}\right\} \rightarrow \mathbb{C}$ via $g_{k}(z):=f_{k}\left(z_{k}+\rho_{k} z\right)$ (indeed, if $|z|<r_{k} / \rho_{k}$, then $\left.\left|z_{k}+\rho_{k} z\right|<\left|z_{k}\right|+r_{k}<\left|\zeta_{k}\right|+2 r_{k}<1\right)$. Note that $r_{k} / \rho_{k}=r_{k} f_{k}^{\sharp}\left(z_{k}\right) \geq r_{k} f_{k}^{\sharp}\left(\zeta_{k}\right) \rightarrow \infty$, so every compact subset of $\mathbb{C}$ is contained in the domain of definition of most $g_{k}$. We have $g_{k}^{\sharp}(0)=\rho_{k} f_{k}^{\sharp}\left(z_{k}\right)=1$.

The $g_{k}$ satisfy, for $|z|<r_{k} / \rho_{k}$,

$$
\begin{aligned}
g_{k}^{\sharp}(z) & =\rho_{k} f_{k}^{\sharp}\left(z_{k}+\rho_{k} z\right) \leq \rho_{k} \frac{M_{k}}{1-\left|z_{k}+\rho_{k} z-\zeta_{k}\right| / r_{k}} \\
& \leq \frac{1-\left|z_{k}-\zeta_{k}\right| / r_{k}}{1-\left|z_{k}-\zeta_{k}\right| / r_{k}-\left|\rho_{k} z\right| / r_{k}}=\frac{1}{1-\frac{\left|\rho_{k} z\right| / r_{k}}{1-\left|z_{k}-\zeta_{k}\right| / r_{k}}} \\
& =\frac{1}{1-\frac{|z|}{r_{k} M_{k}}} .
\end{aligned}
$$

Since $r_{k} M_{k} \geq r_{k} f_{k}^{\sharp}\left(\xi_{k}\right) \rightarrow \infty$, this implies that the $g_{k}^{\sharp}$ are uniformly bounded on compact sets, so they converge subsequentially. Since $g_{k}(0)=f_{k}\left(z_{k}\right) \rightarrow b$, it follows that the $g_{k}$ converge locally uniformly to a holomorphic limit function with $g^{\sharp}(0)=1$, so $g$ is non-constant. But a locally uniform limit of entire functions is entire, hence $b \in \mathbb{C}$.

\section{Theorem 1.20 (Julia and Fatou Sets).}

The Julia set equals the closure of the set of repelling periodic points.

Proof. We give a short proof due to Berteloot and Duval [BD00]. It is clear that the set of repelling periodic points is contained in the Julia set, and thus also their closure.

For the converse, let $P_{0}$ be the countable set of points in the forward orbits of the critical points (we do not take the closure here). Let $P$ equal $P_{0}$ union the set of exceptional points. Let $U$ be any open set in $\mathbb{C}$ that intersects $J(f)$. We will show that $U$ contains a repelling periodic point of $f$. Since $J(f)$ is locally uncountable (Theorem 1.4), we may choose a point $a \in(J(f) \cap U) \backslash P$.

Apply the Zalcmann Lemma 1.19 to the family of functions $f^{\text {on }}$ based at the point $a \in J(f)$ : there exist a sequence $\rho_{k} \rightarrow 0$, a sequence $z_{k} \rightarrow a$, a non-constant entire function $g: \mathbb{C} \rightarrow \mathbb{C}$, and a subsequence $f^{\circ n_{k}}$ so that $g_{k}(\xi):=f^{\circ n_{k}}\left(z_{k}+\rho_{k} \zeta\right) \rightarrow g(\zeta)$ uniformly on compacts. 
We start with the following claim: if there exists a disk $D \subset \mathbb{C}$ so that $g: D \rightarrow$ $g(D)$ is univalent and $a \in g(D) \subset U$, then $f$ has a repelling periodic point in $U$.

To see the claim, define a sequence of contractions $r_{k}(z):=z_{k}+\rho_{k} z$. Then $g_{k}=f^{\circ n_{k}} \circ r_{k}$. Let $D^{\prime}$ be a bounded open disk with $\overline{D^{\prime}} \subset D$ and $a \in g\left(D^{\prime}\right)$. For $k$ sufficiently large, $g_{k}$ is univalent on $D^{\prime}$ and $a \in g_{k}\left(D^{\prime}\right) \subset U$, and also $r_{k}\left(D^{\prime}\right) \subset g_{k}\left(D^{\prime}\right)=$ $f^{\circ n_{k}} \circ r_{k}\left(D^{\prime}\right) \subset U$ (the first assertion follows from the fact that the $r_{k}$ are contractions towards $a$ ). This implies that $\left(f^{\circ n_{k}}\right)^{-1}\left(r_{k}\left(D^{\prime}\right)\right) \subset r_{k}\left(D^{\prime}\right)$, so $\left(f^{\circ n_{k}}\right)^{-1}$ has an attracting fixed point in $r_{k}\left(D^{\prime}\right) \subset U$, by the Schwarz Lemma. This proves the claim.

Our next assertion is that there always exists a disk $D$ as in the claim. Let $V \subset \mathbb{C}$ be the set of points $z$ for which there is a disk $D$ so that $g: D \rightarrow g(D)$ is univalent and $g(D)$ is a neighborhood of $z$. Every point in $\mathbb{C} \backslash V$ is either an omitted value or a critical value of $g$. Since $g$ has at most one omitted value by the Picard theorem and at most countably many critical values, it follows that $\mathbb{C} \backslash V$ is at most countable. Clearly, $V$ is open, and it intersects $J(f)$. We need to show that $a \in V$.

By assumption, the backward orbit of $a$ is infinite. Since $V$ is open and intersects $J(f)$, there is a $b \in V \cap U$ and an $n \in \mathbb{N}$ with $f^{\circ n}(b)=a$. Then $b$ has a neighborhood $W$ for which $f^{\circ n}(W)$ is univalent and contains $a$, so we indeed have $a \in V$, thus proving our assertion.

We have shown that each open set $U$ intersecting $J(f)$ contains a repelling periodic point. Since $U$ is arbitrary, this proves the theorem.

\section{Theorem 1.21 (Periodic Points of Almost All Periods).}

Every entire function has repelling periodic points of all periods, except possibly period 1 .

An example of an entire function without periodic points of period 1 is $z \mapsto e^{z}+z$. Theorem 1.21 was shown by Bergweiler in 1991 [Be91] (even for meromorphic functions), answering a question of Baker from 1960 [Ba60]. A simple proof of the fact that every entire function has periodic points of all periods except possibly 1 can be found in [BB01]. (Already in 1948, Rosenbloom [Ro48] had shown that for every entire function $f$ and every $n \geq 1, f^{\circ n}$ has infinitely many fixed points; these are periodic points of $f$ of period $n$ or dividing $n$.)

Remark 1.22. Meromorphic functions with finitely many poles are often viewed as being similar as entire functions: near $\infty$, both have no poles. However, there are significant differences: for instance, periodic Fatou components of entire functions are simply connected (Theorem 2.5), but this is not true for meromorphic functions.

\section{The Fatou Set of Entire Functions}

In this section, we describe the possible types of components of the Fatou set of an entire function. These types are similar as those for polynomials, but there are two extra types: Baker domains (domains at infinity) and wandering domains. We give examples for both. 


\section{Theorem 2.1 (Classification of Fatou Components).}

Any Fatou component has exactly one of the following types:

- a periodic component in which the dynamics converges to an attracting or superattracting cycle;

- a periodic component in which the dynamics converges to a parabolic cycle;

- a periodic component in which the dynamics is conformally conjugate to an irrational rotation on a disk (a Siegel disk);

- a periodic component in which the dynamics converges to $\infty$ (a Baker domain);

- a preperiodic component that eventually maps to a periodic component in one of the types above;

- a non-periodic component for which all forward iterates are disjoint (a wandering domain).

Remark 2.2. The difference to the polynomial case is the possibility of Baker domains (these are similar to parabolic domains in the sense that the dynamics converges locally uniformly to a boundary point, except that the parabolic boundary point is replaced by the essential singularity at $\infty$ ) and of wandering domains. Rational and meromorphic maps may also have Arnol'd-Herman rings, but these do not exist for entire maps (by the maximum principle).

The proof of the classification of periodic Fatou components [BKL91,EL89] is similar as for rational maps (see Milnor [Mi06]). A difference occurs for Fatou components in which some and thus all orbits converge to the boundary: if $f$ is defined in a neighborhood of a limiting boundary point, then this boundary point must be parabolic; if not, then this boundary point is the essential singularity at $\infty$ and we have a Baker domain. The only other difference is the possibility that a Fatou component may not be eventually periodic (i.e., a wandering domain): for rational maps, this is ruled out by Sullivan's theorem, but wandering domains do occur for transcendental maps (see Examples 2.10 and 2.11 below). However, there are some families of transcendental entire functions without wandering domains: for instance, see Theorem 3.4 and [EL89, Be93, BHKMT93]. In particular, [BHKMT93] discusses the non-existence of wandering domains in certain cases without using quasiconformal methods: they show that all limit functions of wandering domains must be limit points of the set of singular orbits.

\section{Theorem 2.3 (Singular Values and Fatou Components).}

Every cycle of attracting and parabolic Fatou components contains a singular value; and every boundary point of every Siegel disks is a limit point of postsingular points.

The proof of this result is the same as in the rational case [Mi06].

Remark 2.4. The connectivity of a Fatou component $U$ is the number of connected components of $\overline{\mathbb{C}} \backslash U$ (it may be $\infty$ ). It is an easy consequence of the RiemannHurwitz formula that the connectivity of $f(U)$ is at most that of $U$. Therefore, every wandering domain has eventually constant connectivity (finite or infinite). Since $f$ has no poles, the image of a multiply connected Fatou component is multiply connected. 
If the connectivity of $U$ is finite at least 3 and does not decrease, then by the Riemann-Hurwitz formula $U$ maps homeomorphically onto its image, and the same is then true for the bounded complementary domains of $U$; this is a contradiction. Therefore, every multiply connected Fatou component is either infinitely connected or eventually doubly connected.

\section{Theorem 2.5 (Multiply Connected Fatou Component).}

Every multiply connected Fatou component $U$ of an entire transcendental function has the following properties:

- $U$ is a wandering domain;

- $U$ is bounded;

- U has eventual connectivity 2 or $\infty$;

- the iterates converge to $\infty$ uniformly;

- each compact $K \subset \mathbb{C}$ is separated from $\infty$ by all but finitely many of the iterated images of $U$;

- the map from each component on the orbit of $U$ to its image has a finite mapping degree, and this degree tends to $\infty$ under iteration;

- the $f^{\circ n}(U)$ contain annuli with moduli tending to $\infty$.

Remark 2.6. A Fatou component satisfying the properties of Theorem 2.5 is sometimes called a Baker wandering domain (caution: this notion must not be confused with that of a Baker domain: a Baker domain is a periodic Fatou component in which the iterates tend to $\infty)$.

Simple connectivity of periodic Fatou components was observed by Baker in [Ba84] (see also Töpfer [Tö39]); the description of multiply connected wandering domains is from [Ba76]. See also [Ri08,Zh06].

Sketch of proof. Let $U$ be a multiply connected Fatou component and let $\gamma_{0} \subset U$ be a simple closed curve that is non-contractible. Let $U_{0}$ be the bounded domain surrounded by $\gamma_{0}$. Inductively, let $U_{n+1}$ be the largest open domain with $\partial U_{n+1} \subset$ $f\left(\gamma_{n}\right)$ and let $\gamma_{n+1}:=\partial U_{n+1}$ (if $V_{n+1}$ is the unique unbounded component of $\mathbb{C} \backslash$ $f\left(\gamma_{n}\right)$, then $\left.U_{n+1}:=\mathbb{C} \backslash \overline{V_{n+1}}\right)$. By the maximum principle, it follows that $f\left(U_{n}\right) \subset$ $U_{n+1}$.

The set $U_{0}$ intersects the Julia set and hence contains periodic points (Theorem 1.20). Replacing $f$ by an iterate, we may suppose that $U_{0}$ contains a fixed point $p$. Therefore, all $\gamma_{n}$ separate $p$ from $\infty$. Denote the hyperbolic length of $\gamma_{n}$ within $U_{n}$ by $\ell\left(\gamma_{n}\right)$. Then clearly $\ell\left(\gamma_{n+1}\right) \leq \ell\left(\gamma_{n}\right)$ for all $n$.

If $U$ contains a fixed point, then all $\gamma_{n}$ have uniformly bounded hyperbolic distances in $U$ from this fixed point, and hence the $f^{\circ n}$ are uniformly bounded on $U_{0}$, so $\gamma_{0}$ was contractible in $U$ contrary to the hypothesis. A similar argument applies if $U$ contains any point with bounded orbit, in particular if $U$ contains a periodic point or if $U$ is a parabolic domain, so we are left with the cases of Baker domains and wandering domains.

Now suppose that $U$ is a periodic Baker domain, say of period 1 ; then $\gamma_{n} \rightarrow \infty$ uniformly. Moreover, the winding number of $f\left(\gamma_{n}\right)$ around $p$ tends to $\infty$. This implies 
that each $f\left(\gamma_{n}\right)$ contains a simple closed subcurve, say $\hat{\gamma}_{n+1}$, that surrounds $p$ and with hyperbolic length within $U$ tending to 0 . By the Collaring Theorem [DH93], it follows that $U$ contains round annuli, say $A_{n}$, that contain $\hat{\gamma}_{n}$ and with moduli $\bmod A_{n} \asymp 1 / \ell\left(\hat{\gamma}_{n}\right)$. Since the Julia set is unbounded, there must be infinitely many $n$ for which $A_{n}$ and $A_{n+1}$ are non-homotopic in $U$ and so that there is an $a_{n} \in J(f)$ that is surrounded by $A_{n+1}$ but not by $A_{n}$. As the moduli of $A_{n}$ and $A_{n+1}$ become large, the hyperbolic distances in $U$ between points in $\hat{\gamma}_{n}$ and in $\hat{\gamma}_{n+1}$ must be unbounded (these distances exceed the distances in $\mathbb{C} \backslash\left\{0, a_{n}\right\}$, which after rescaling is equivalent to $\mathbb{C} \backslash\{0,1\}$, and the annuli $A_{n}, A_{n+1}$ rescale to essential annuli in $\mathbb{C} \backslash\{0\}$ with large moduli so that exactly one of them surrounds 1 ). However, the lengths of $\hat{\gamma}_{n}$ tend to zero, while the distances between $\hat{\gamma}_{n}$ and $\hat{\gamma}_{n+1}$ are bounded with respect to the hyperbolic metric in $U$. This contradiction shows that periodic Baker domains are simply connected.

Finally, we discuss the case that $U$ is a wandering domain. In this case, all $\gamma_{n}$ must be disjoint, and no $\gamma_{n}$ can be contained in $\gamma_{m}$ for $m<n$ (or the entire domain $U_{n}$ would converge to an attracting periodic point, so $\gamma_{n}$ would be contractible). Since $f\left(U_{n}\right) \subset U_{n+1}$, it follows that $\gamma_{n+1}$ separates $\gamma_{n}$ from $\infty$. The argument now continues as above.

\section{Corollary 2.7 (Asymptotic Values and Multiple Connectivity).}

If an entire function has a finite asymptotic value, then all Fatou components are simply connected.

\section{Example 2.8 (A Baker Domain).}

The map $f(z)=z+1+e^{-z}$ has a Baker domain containing the right half plane.

Clearly, each $z \in \mathbb{C}$ with $\operatorname{Re} z>0$ has $\operatorname{Re} f(z)-\operatorname{Re} z>0$, and this quantity is bounded below uniformly in each half plane $\operatorname{Re} z>\delta>0$. The Fatou set $F(f)$ is connected and contains the right half plane $\mathbb{H}:=\{z \in \mathbb{C}: \operatorname{Re} z>0\}$ : we have $F(f)=\bigcup_{n>0} f^{-n}(\mathbb{H})$, and the Julia set is an uncountable collection of curves (see the discussion of Cantor Bouquets in Section 4). This example is due to Fatou [Fa26].

Many more results on Baker domains can be found in the recent survey article [Ri08] by Rippon.

\section{Example 2.9 (A Wandering Domain).}

The map $f(z)=z-1+e^{-z}+2 \pi i$ has a wandering domain.

This example is due to Herman from around 1985. The map $N: z \mapsto z-1+e^{-z}$ is the Newton map for the function $z \mapsto e^{z}-1$. The basin of the root $z=0$ is clearly invariant under $N$, and the basins of the roots $z=2 \pi i n$ for $n \in \mathbb{Z}$ are translates of the basin of $z=0$ by $2 \pi i n$. We have $N(z+2 \pi i)=N(z)+2 \pi i$, so the Fatou and Julia sets are $2 \pi i$-invariant. Now $f(z)=N(z)+2 \pi i$, and $f$ preserves $F(N)$ and $J(N)$, so $F(f) \supset F(N)$ by Montel's Theorem A.2. Suppose there was a point $z \in$ $F(f) \cap J(N)$. Let $U_{0} \subset F(f)$ be a neighborhood of $z$. Arbitrarily close to $z$, there are points converging under $N$ to basins of different roots (Theorem 1.7). This would imply that $F(f)$ was connected, but this leads to the following contradiction: initial 
points in $U_{0}$ at arbitrarily short hyperbolic distance within $F(f)$ would have orbits at least $2 \pi$ apart, in contradiction to the fact that hyperbolic distances never increase. Therefore, $F(f)=F(N)$. Therefore, all basins of roots for the Newton map $N$ move a distance $2 \pi i$ in each iteration of $f$, so they turn into wandering domains for $f$.

\section{Example 2.10 (A Simply Connected Wandering Domain).}

The map $f(z)=z+\sin z+2 \pi$ has a bounded simply connected wandering domain.

This example is due to [Ba84] (more generally, he considers functions of the type $z \mapsto z+h(z)$, where $h$ is a periodic function). In order to describe this example, first observe that for $g(z)=z+\sin z$, all points $n \pi$ (for odd integers $n$ ) are superattracting fixed points, so their immediate basins are disjoint. Moreover, $g(z+2 \pi)=g(z)+2 \pi$, so the Julia set is $2 \pi$-periodic, and as above $J(f)=J(g)$. Therefore, $f$ maps the immediate basin of $n \pi$ to the immediate basin of $(n+2) \pi$ for odd $n$, and this is a wandering domain for $f$. All critical points of $g$ are superattracting fixed points, so it follows easily that these basins are simply connected. It is not hard to see that these basins of $g$ are bounded (the imaginary axis is preserved under $g$ and all points other than zero converge to $\infty$, and the same is true for $2 \pi$-translates, so every Fatou component has bounded real parts, and points with large imaginary parts have images with much larger absolute values).

\section{Example 2.11 (A Baker Wandering Domain).}

For appropriate values of $c>0$ and $r_{n} \rightarrow \infty$, the map

$$
f(z)=c z^{2} \prod_{n \geq 1}\left(1+z / r_{n}\right)
$$

has a multiply connected wandering domain.

This is the original example from Baker [Ba63, Ba76]. The idea is the following: the radii $r_{n}$ grow to $\infty$ very fast. There are annuli $A_{n}$ with large moduli containing points $z$ with $r_{n} \ll|z| \ll r_{n+1}$. On them, the factors $\left(1+z / r_{m}\right)$ with $m>n$ are very close to 1 so that even their infinite product can be ignored, while the finitely many bounded factors essentially equal $z / r_{n}$, so on the annuli $A_{n}$ the map $f(z)$ takes the form $c_{n} z^{n+2}$. The factors are arranged so that $f$ sends $A_{n}$ into $A_{n+1}$ with degree $n+2$. Therefore all points in $A_{n}$ escape to $\infty$. The point $z=0$ is a superattracting fixed point. The fact that the $A_{n}$ are contained in a (Baker) wandering domain, rather than a (periodic) Baker domain (basin at $\infty$ ) follows from simple connectivity of Baker domains (Theorem 2.5).

Remark 2.12. Baker wandering domains may have any connectivity, finite or infinite [KS08] (the eventual connectivity will be 2 or $\infty$ ). These may coexist with simply connected wandering domains [Be]. These examples use a modified construction from Example 2.11: essentially, there is a sequence of radii $0<\cdots<r_{n}<R_{n}<$ $r_{n+1}<\ldots$ and concentric annuli $A_{n}:=\left\{z \in \mathbb{C}: r_{n}<|z|<R_{n}\right\}, B_{n}:=\left\{z \in \mathbb{C}: R_{n}<\right.$ $\left.|z|<r_{n+1}\right\}$. On $B_{n}$, we define the map $g(z)=z^{n+1}\left(1+z / \sqrt{R_{n} r_{n+1}}\right)$, which is very close to $z^{n+1}$ near the inner boundary, and to $z^{n+2}$ near the outer. On the annuli 
$A_{n}$, we use a $C^{\infty}$ map that is close to a holomorphic map (quasiregular with small dilatation), and arrange things again so that $f\left(A_{n}\right) \subset A_{n+1}$ so that $g$ sends $A_{n}$ to its image as a branched cover of degree $n+2$. Using the Measurable Riemann Mapping Theorem, it follows that this $C^{\infty}$-map $g$ is quasiconformally conjugate to an entire holomorphic map $f$ with similar properties, in particular with a wandering annulus. The construction using quasiconformal surgery gives greater flexibility and allows one to determine the dynamical properties of these maps (for instance, in Baker's example it was not known whether the Baker wandering domain was eventually doubly or infinitely connected).

Kisaka and Shishikura show that every point in the Fatou component of $A_{n}$ lands in $A_{N}$ for $N>n$ large enough, and use this to conclude that the Fatou component of $A_{n}$ is doubly connected. Modifying the construction for small values of $n$, they obtain Baker wandering domains with any finite, or with infinite connectivity (as well as with many further properties; see [KS08,Be]).

There may even be infinitely many disjoint orbits of wandering domains [Ba84, RS99a].

\section{Entire Functions of Finite Type and of Bounded Type}

The set of all entire functions is extremely large and accommodates a huge amount of dynamical variety, so in order to have good control and to obtain strong results it is often useful to restrict to smaller classes of functions.

\section{Definition 3.1 (Special Classes of Entire Functions).}

The class $S$ ("Speiser class") consists of those entire functions that have only finitely many singular values. The class $B$ ("the Eremenko-Lyubich class of functions of bounded type") consists of those entire functions for which all singular values are contained in a bounded set in $\mathbb{C}$.

Remark 3.2. For an entire function of class $B$, all transcendental singularities over $\infty$ are logarithmic.

Remark 3.3. An entire function $f$ has finitely many critical points and finitely many asymptotic tracts if and only if $f$ has the form $f(z)=c+\int P\left(z^{\prime}\right) \exp \left(Q\left(z^{\prime}\right)\right) d z^{\prime}$ for polynomials $P, Q$.

\section{Theorem 3.4 (Fatou Components for Class $S$ and Class $B$ ).}

A function of class $S$ does not have Baker domains or wandering domains. A function of class $B$ does not have Baker domains, and it does not have wandering domains in which the dynamics converges to $\infty$.

Remark 3.5. This result is due to Eremenko and Lyubich [EL84b,EL92]; for wandering domains in class $S$, see also Goldberg and Keen [GK86]. The proof is outlined below. 


\section{Theorem 3.6 (The Fatou-Shishikura-Inequality).}

A function of class $S$ has no more non-repelling periodic cycles than singular grand orbits (and in particular, no more than singular values).

Remark 3.7. A singular grand orbit is any set of singular values for which their orbits intersect pairwise. Theorem 3.6 is due to Eremenko and Lyubich [EL89]; compare also Shishikura's proof of the Fatou-Shishikura-inequality [Sh87]. A different proof is due to Epstein [Ep].

\section{Definition 3.8 (Logarithmic Tract).}

For an entire function $f \in B$, a logarithmic tract $T$ is a connected component of $f^{-1}\left(\mathbb{C} \backslash D_{R}\right)$, where $D_{R} \subset \mathbb{C}$ is the closed disk around 0 of radius $R>0$, and $R$ is chosen so that $D_{R}$ contains all singular values of $f$ as well as $f(0)$.

Remark 3.9. By construction, the restriction $f: T \rightarrow \mathbb{C} \backslash D_{R}$ is a covering. For transcendental entire functions, it is a universal covering: otherwise, the mapping degree from $T$ to $\mathbb{C} \backslash D_{R}$ would be finite and $\partial T$ would be a circle, and by the maximum modulus principle this would imply that $T$ contained a neighborhood of $\infty$, so $f$ would be a polynomial.

For a transcendental entire function, the number of logarithmic tracts may be finite or infinite. A function of finite order has only finitely many tracts by DenjoyCarleman-Ahlfors-theorem (Theorem 1.17). The condition $|f(0)|<R$ is required for the construction of logarithmic coordinates as described below.

\section{Definition 3.10 (Logarithmic Coordinates).}

For a function $f \in B$, a corresponding function in logarithmic coordinates is defined as follows: let $T_{j}$ be the logarithmic tracts of $f$ and let $T_{j, k}^{\prime}$ be the components of $\log \left(T_{j}\right)$ (so that $\exp \left(T_{j, k}^{\prime}\right)=T_{j}$ and $T_{j, k+1}^{\prime}=T_{j, k}^{\prime}+2 \pi i$ ). Then a function $F$ corresponding to $f$ in logarithmic coordinates is any holomorphic function

$$
F: \bigcup_{j, k} T_{j, k}^{\prime} \rightarrow H_{\log R}:=\{z \in \mathbb{C}: \operatorname{Re} z>\log R\}
$$

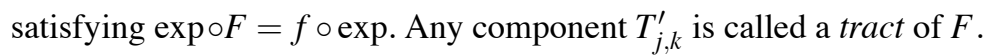

Remark 3.11. Since exp: $H_{\log R} \rightarrow \mathbb{C} \backslash D_{R}$ and $f: T_{j} \rightarrow \mathbb{C} \backslash D_{R}$ are universal covers, while exp: $T_{j, k}^{\prime} \rightarrow T_{j}$ is a conformal isomorphism, it follows that each restriction $F: T_{j, k}^{\prime} \rightarrow H_{\log R}$ is also a conformal isomorphism. Note that $F$ is determined by $f$ only up to additive integer multiplies of $2 \pi i$, separately for each tract (in general, $F$ is not defined on any right half plane, or on any connected set containing all the tracts).

Entire functions of bounded type are defined so that logarithmic coordinates exist outside any disk $D_{R}$ that is large enough so as to contain all singular values and the point $f(0)$ (the latter condition is needed in order to assure that $T_{j} \not \supset 0$, so logarithms can be taken on all tracts). Logarithmic coordinates were introduced to transcendental dynamics by Eremenko and Lyubich [EL84a,EL84b, EL92]. 
The following fundamental estimate of Eremenko and Lyubich [EL92, Lemma 1] is very useful. Write $\mathbb{H}:=\{z \in \mathbb{C}: \operatorname{Re} z>0\}$ for the right half plane.

Lemma 3.12 (Expansion on Logarithmic Tracts). If $T^{\prime}$ is a logarithmic tract and $F: T^{\prime} \rightarrow \mathbb{H}$ is a conformal isomorphism, then

$$
\left|F^{\prime}(z)\right| \geq \frac{1}{4 \pi} \operatorname{Re} F(z) .
$$

Remark 3.13. All we are using about $T^{\prime}$ is that it is simply connected and disjoint from $T^{\prime}+2 \pi i k$ for $k \in \mathbb{Z} \backslash\{0\}$.

Proof. Since $F: T^{\prime} \rightarrow \mathbb{H}$ is a conformal isomorphism, it has a conformal inverse $G: \mathbb{H} \rightarrow T^{\prime}$. Let $D$ be the open disk around $F(z)$ with radius $R=\operatorname{Re} F(z)$. Then by the Koebe 1/4-theorem, $G(D)$ contains a disk around $G(F(z))=z$ of radius $R\left|G^{\prime}(F(z))\right| / 4=\operatorname{Re} F(z) / 4\left|F^{\prime}(z)\right|$; but by periodicity of the tracts in the vertical direction, the image radius must be at most $\pi$, and (1) follows.

Sketch of proof of Theorem 3.4. For a function $F: \cup T_{j, k}^{\prime} \rightarrow \mathbb{H}$ in logarithmic coordinates, we claim that the set of points $z$ with $\operatorname{Re} F^{\circ n}(z) \rightarrow \infty$ has no interior (here we assume for simplicity that, possibly by translating coordinates, $F\left(T_{j, k}^{\prime}\right)=\mathbb{H}$ for all tracts $T_{j, k}^{\prime}$ ). The key to this is Lemma 3.12. Suppose that $F: \cup T_{j, k}^{\prime} \rightarrow \mathbb{H}$ has an open set in the escaping set, say the round disk $D_{\varepsilon}(z)$ around some point $z$ with $\operatorname{Re} F^{\circ n}(z) \rightarrow \infty$. Then (1) implies $\left|F^{\prime}\left(F^{\circ n}(z)\right)\right| \rightarrow \infty$, hence $\left|\left(F^{\circ n}\right)^{\prime}(z)\right| \rightarrow \infty$. Using the Koebe $1 / 4$ theorem it follows that $I(F)$ contains disks of radii $\varepsilon\left|\left(F^{\circ n}\right)^{\prime}(z)\right| / 4$. Since these radii must be bounded by $\pi$, it follows that $\varepsilon=0$. This proves the claim, and this implies that for functions $f \in B$, the escaping set $I(f)$ has empty interior. This proves Theorem 3.4, except for the fact that functions $F \in S$ do not have wandering domains. We do not describe this proof here. It uses the essential ideas of Sullivan's proof in the rational case (see [Mi06]), in particular quasiconformal deformations, and depends on the fact that small perturbations of functions in class $S$ live in a finite-dimensional space.

\section{Corollary 3.14 (Entire Functions with Empty Fatou Set).}

If an entire function $f$ in class $B$ has the property that all its singular values are strictly preperiodic or escape to $\infty$, then $f$ has empty Fatou set.

Indeed, any Fatou component of $f$ would require an orbit of a singular value that converges to an attracting or parabolic periodic orbit, or accumulates on the boundary of a Siegel disk, by Theorems 2.3 and 3.4.

Functions $f \in B$ have a most useful built-in partition with respect to which symbolic dynamics can be defined for those orbits that stay sufficiently far away from the origin.

\section{Definition 3.15 (Symbolic Dynamics and External Address).}

Consider a function $f \in B$ and let $R>0$ be large enough so that $D_{R}(0)$ contains all singular values as well as $f(0)$, and let $F: T_{j, k}^{\prime} \rightarrow H_{\log R}$ be a function in logarithmic coordinates corresponding to $f$. Here $k$ ranges through the integers so that $T_{j, k+1}^{\prime}=$ $T_{j, k}^{\prime}+2 \pi i$, and $j$ ranges through some (finite or countable) index set $J$, say. 
The external address of a point $z \in \mathbb{C}$ so that $F^{\circ n}(z) \in \bigcup_{j, k} T_{j, k}^{\prime}$ for all $n \geq 0$ is the sequence $(j, k)_{n}$ so that $f^{\circ n}(z) \in T_{(j, k)_{n}}^{\prime}$ for all $n$.

Our "external address" is an "itinerary" with respect to the partition given by the $T_{j, k}^{\prime}$; however, in order to achieve notational consistency, we prefer to use the term "itinerary" in a different context: the analogy are dynamic rays of polynomials, our external addresses correspond to external angles (or their expansion in base $d$, the degree of the polynomials); in many cases, one can define itineraries of dynamic rays, so that different dynamic rays have the same itinerary if and only these two rays land at a common point. See the discussion in [SZ03b, Section 4.5] or [BS].

If $\underline{s}=(j, k)_{n}$ is an external address, define

$$
J_{\underline{s}}:=\{z \in \mathbb{C}: z \text { has external address } \underline{s}\} .
$$

All sets $J_{\underline{\underline{s}}}$ are obviously disjoint. Each set $J_{\underline{\underline{s}}}$ is a closed set, either empty or unbounded: $J_{\underline{s}} \cup\{\infty\}$ is the nested intersection of the non-empty compact sets $\left\{z \in \mathbb{C}: f^{\circ n}(z) \in T_{(j, k)_{n}}^{\prime}\right.$ for $n=0,1, \ldots, N$ and $\left.N \in \mathbb{N}\right\}$, so $J_{\underline{s}} \cup\{\infty\}$ is compact and non-empty.

An important special case that simplifies the situation occurs if all $T_{j, k}^{\prime} \subset H_{\log R}$; this occurs if the original entire function $f$ has an attracting fixed point that attracts all singular values of $f$. In this case, all $J_{\underline{s}} \cup\{\infty\}$ are nested intersections of non-empty compact and connected sets, hence compact and connected. If the tract boundaries are sufficiently well-behaved (for instances, so that they are eventually parametrized by their real parts, or more generally that their boundaries do not "wiggle" too much), then it can be shown that each non-empty $J_{\underline{s}}$ is a curve that connects some point $z \in \mathbb{C}$ to $\infty$. These conditions are satisfied in particular when $f$ has finite order in which all singular values are attracted by the same fixed point (see Barański [Bar07]). A prototypical situation in which this had been considered relatively early is the case of exponential maps with attracting fixed points; consider [DK84]. More general results showing that the $J_{\underline{s}}$ are curves, and also providing counterexamples in other cases, are established in [RRRS]. In many cases, all but possibly one point in $J_{\underline{s}}$ are escaping points; a frequent concept that occurs in this case is that of a Cantor bouquet. We will discuss this situation in more detail in Section 4. However, there are entire functions of bounded type, even hyperbolic ones, for which the Julia set does not contain curves. An example of this was constructed in [RRRS].

\section{Theorem 3.16 (Non-Existence of Curves).}

There are hyperbolic entire functions of bounded type for which every path component of $J(f)$ is bounded (or even a point).

Here, we give a few ideas of the arguments in Theorems 3.16. The work takes place in logarithmic coordinates and consists of two steps: first we construct a domain $T \subset \mathbb{H}$ with a conformal isomorphism $G: T \rightarrow \mathbb{H}$ sending the boundary point $\infty \in \partial T$ to the boundary point $\infty \in \partial \mathbb{H}$ and so that all path components of

$$
J(G):=\{z \in T: G \text { can be iterated infinitely often starting at } z\}
$$




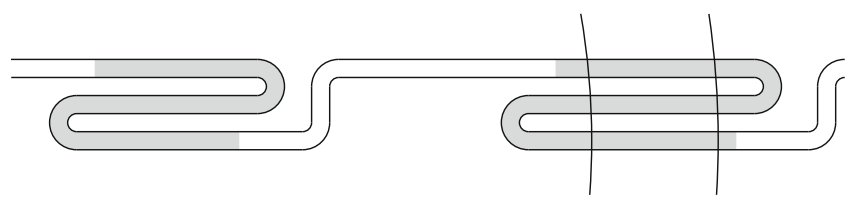

Fig. 1 The wiggles in the proof of Theorem 3.16. Shown is (part of) the tract $T$ with two wiggles (the tract extends to the right towards infinity and has infinitely many wiggles). The shaded domain in the left wiggle maps to a large half annulus that is indicated by two circular arcs, and this image annulus must intersect the second wiggle so that each curve through the second wiggle intersects the image annulus three times

are bounded. Then an approximation method is used to show that there exists an entire function of bounded type $f$ that yields a function $F$ in logarithmic coordinates that is close enough to $G$ so as to have similar properties.

For the first step, the domain $T$ is a long sequence of wiggles as indicated in Figure 1: there are two real parts $x_{1}<y_{1}$ so that $T$ transverses the interval of real parts in $\left[x_{1}, y_{1}\right]$ three times; this part of $T$ is called the first wiggle. The second wiggle of $T$ is such that $G^{-1}(T)$ sends the second wiggle into the first one so that $G^{-1}(T)$ has to transverse the interval $\left[x_{1}, y_{1}\right]$ nine times. The third wiggle has the property that its $G$-preimage in $T$ "wiggles" three times through the second wiggle, and the second preimage wiggles nine times through the first. Iterating this argument, it follows that any curve in $J(G)=\bigcap_{n>0}\left(G^{-1}\right)^{\circ n}(T)$ must connect real parts $x_{1}$ and $y_{1}$ infinitely often in alternating order, and this is impossible. Therefore, points in $J(G)$ to the left of the first wiggle have bounded path components. Similarly, it follows that no path component within $J(G)$ can traverse any of the infinitely many wiggles in $T$ further to the right.

The second step in the construction consists of finding an entire function $f$ that has a map $F$ in logarithmic coordinates close to $G$. This is a classical approximation procedure (see for instance [GE79]). A description of this method can be also found in [RRRS].

The "size" of the wiggles must be large, in the sense that the ratio $x_{2} / x_{1}$ between the ends of the wiggles must tend to $\infty$. To see this, we decompose $G=$ $G_{2} \circ G_{1}$, where $S=\{z \in \mathbb{C}: \operatorname{Re} z>0,|\operatorname{Im} z|<\pi / 2\}$ is a one-sided infinite strip and $G_{1}: T \rightarrow S, G_{2}: S \rightarrow \mathbb{H}$ are conformal isomorphisms fixing the boundary points $\infty$. Then $G_{2}$ is essentially the exponential map, except near the left end, and $G_{1}$ can be thought of as an affine map along the long straight pieces of $T$, and just "bending" the tract $T$ straight into $S$ near the turns.

If a wiggle satisfies $y_{n} / x_{n}<\kappa$, then the log of this wiggle (i.e., its preimage under $G_{2}$ ) has length at most $\log \kappa$, and the same is true for the $G$-preimage. After the next iterated preimage, a wiggle of bounded length at large real part becomes small, and we do not get bounded path components. Therefore, $y_{n} / x_{n} \rightarrow \infty$ (even extremely fast) is necessary for counterexamples. This implies fast growth of $G$ : if $y_{n} / x_{n}$ is large, that means that the tract $T$ bends back far to the left (it has big "wiggles"), and therefore $T$ contains points that are (conformally) far out in $T$ (so that $\operatorname{Re} F(z$ ) is large), but $\operatorname{Re} z$ is small. 
This condition is related to finite order as follows: if an entire function $f$ has finite order as in Definition 1.15, then the corresponding function in logarithmic coordinates satisfies

$$
\lim \sup _{\operatorname{Re} z \rightarrow+\infty} \log \operatorname{Re} F(z) / \operatorname{Re} z<\infty .
$$

We thus use (2) as a definition for a function in logarithmic coordinates to have finite order. Geometric function theory thus implies that large wiggles entail fast growth of $F$, and this is restricted if $F$ or equivalently $f$ have finite order.

\section{The Escaping Set}

A significant amount of work on the dynamics of an entire function $f$ has been, and still is, concerned with the set $I(f)$ of escaping points. There are several reasons why the escaping set is interesting:

- $I(f)$ is a non-empty invariant set (and unlike the Julia set, it is always a proper subset of $\mathbb{C}$ ).

- The set $I(f)$ often has useful structure, such as the union of curves to $\infty$ ("dynamic rays" and "Cantor Bouquets").

- In many cases, several curves in $I(f)$ connect the same point $z \in \mathbb{C}$ to infinity, even if $z \notin I(f)$ (in other words, several dynamic rays land at a common point). This gives additional structure to the dynamic plane, such as in the famous "puzzle theory" of polynomials [Sch07b, RSch08, Ben].

- $I(f)$ is a subset of the dynamical plane that is often easy to control; for functions of bounded type, it is a subset of the Julia set and thus makes it possible for instance to give lower bounds on the Hausdorff dimension of the Julia set (see Section 5).

- For polynomials, especially with non-escaping critical orbits, $I(f)$ has a very simple structure, and $J(f)=\partial I(f)$; so many descriptions of $J(f)$ for polynomials are based on $I(f)$, and analogous approaches are desired also for entire functions.

While many discussions in the earlier sections hold more generally for iterated meromorphic functions, the discussion of the escaping set (and of functions of bounded type) make sense only for entire functions, in the same sense the polynomial dynamics is much easier to describe than the dynamics of rational mappings.

The fundamental results about the escaping set are due to Eremenko [Er89] (see also Domínguez [Do98]). He established in particular the following.

\section{Theorem 4.1 (The Escaping Set).}

Every transcendental entire function $f$ has the following properties:

- $I(f) \cap J(f) \neq \emptyset$;

- $J(f)=\partial I(f)$;

- all components of $\overline{I(f)}$ are unbounded. 
Examples of Fatou components in $I(f)$ have been given in Examples 2.8 (a Baker domain), 2.9, and 2.10 (wandering domains).

We would like to review especially Fatou's Example 2.9 with $f(z)=z+1+e^{-z}$. There is a single Fatou component, it contains the right half plane, and in it the orbits converge quite slowly to $\infty$. However, for integers $k$, points on $i(2 k+1) \pi+\mathbb{R}^{-}$ converge very quickly to $\infty$ (at the speed of iterated exponentials); these are countably many curves in $I(f)$ (dynamic rays), and the backwards orbits of these curves form many more curves in $I(f)$. In fact, $I(f)$ contains uncountably many curves to $\infty$ (a Cantor bouquet).

Eremenko [Er89] raised some fundamental questions on $I(f)$ that inspired further research.

Question 4.2 (Eremenko's Questions on $I(f)$ ).

Weak version Is every component of $I(f)$ unbounded?

Strong version Can every $z \in I(f)$ be connected to $\infty$ within $I(f)$ ?

These questions have inspired a substantial amount of work, and are often referred to as Eremenko's conjecture (in its weak and strong form). There are various partial results on them, positive and negative. Rippon and Stallard [RS05a, RS05b] showed the following.

\section{Theorem 4.3 (Baker Wandering Domains and $I(f)$ ).}

Let $f$ be an entire function. Then $I(f)$ always has an unbounded component. If $f$ has a Baker wandering domain, then $I(f)$ is connected.

These results are based on a study of the set $A(f)$ as described below. Rempe [Re07] established sufficient conditions for the weak version of Eremenko's question, as follows.

\section{Theorem 4.4 (Unbounded Components of $I(f)$ ).}

If $f$ is an entire function of bounded type for which all singular orbits are bounded, then every component of $I(f)$ is unbounded.

Substantial attention has been given to the speed of escape for points in $I(f)$. A classical lemma, due to Baker [Ba81], is the following.

\section{Lemma 4.5 (Homogeneous Speed of Escape in Fatou Set).}

If $z, w \in I(f)$ are two escaping points from the same Fatou component of an entire function $f$, then $\log \left|f^{\circ n}(z)\right| / \log \left|f^{\circ n}(w)\right|$ is bounded as $n \rightarrow \infty$. If $z$ is in a (periodic) Baker domain, then $\log \left|f^{\circ n}(z)\right|=O(n)$.

This result follows from the observation that the hyperbolic distance between $w$ and $z$ in the hyperbolic metric of $F(f)$ cannot increase, and that this distance is essentially bounded below by the hyperbolic distance of $\mathbb{C} \backslash \mathbb{R}_{0}^{-}$.

Unlike other types of periodic Fatou components, Baker domains need not contain singular values. However, if a Baker domain exists, the set of singular values must be unbounded by Theorem 3.4; there is a stronger result by Bargmann [Ba01] 
and Rippon and Stallard [RS99b] saying that, for every entire function with a Baker domain, there exists a constant $C>0$ so that every annulus $\{z \in \mathbb{C}: r / C<|z|<r C\}$ (for $r$ sufficiently large) intersects the set of singular values of $f$. More results on Baker domains, including classification results, as well as examples of entire functions with infinitely many Baker domains, can be found in Rippon's survey [Ri08].

There are a number of further interesting subsets of $I(f)$ defined in terms of their speed of escape.

Definition 4.6 (The Sets $A(f), L(f)$ and $Z(f)$ ).

For an entire function $f$, the set $A(f)$ is defined as follows: given a large radius $R=R_{0}>0$, define recursively $R_{n+1}:=M\left(R_{n}, f\right)$ and set

$$
\begin{gathered}
A_{R}(f):=\left\{z \in \mathbb{C}:\left|f^{\circ n}(z)\right| \geq R_{n} \text { for all } n \geq 0\right\}, \\
A(f):=\bigcup_{n \geq 0} f^{-n}\left(A_{R}(f)\right) .
\end{gathered}
$$

The set $Z(f)$ is defined as

$$
Z(f):=\left\{z \in \mathbb{C}: \frac{\log \log \left|f^{\circ n}(z)\right|}{n} \rightarrow \infty \text { as } n \rightarrow \infty\right\} .
$$

The set $L(f)$ is defined as

$$
L(f):=\left\{z \in I(f): \limsup \frac{\log \left|f^{\circ n}(z)\right|}{n}<\infty\right\} .
$$

The set $A(f)$ describes essentially those points that escape to $\infty$ as fast as possible; it has been introduced by Bergweiler and Hinkkanen [BH99]. It is quite easy to see that it does not depend on $R$ provided $R$ is large enough so that $J(f) \cap D_{R}(0) \neq \emptyset$. The set $Z(f)$ describes those points that "zip" to $\infty$ much faster than what is possible for polynomials (for the latter, $\log \log \left|f^{\circ n}\right|=O(n)$ ). The set $L(f)$ describes slow escape (the letter $L$ stands for sLow or the German Langsam). These sets are among those introduced by Rippon and Stallard; they are well on their way towards exhausting the alphabet with interesting sets of different speeds of escape. Unfortunately, the speed of escape is not compatible with the alphabetic order of their letters. The following is a subset of what is known on these sets.

\section{Theorem 4.7 (Speed of Escape).}

For every transcendental entire function $f$, the sets $A(f), L(f)$ and $Z(f)$ satisfy the following:

1. $J(f) \cap A(f) \neq \emptyset$;

2. $A(f) \subset Z(f)$, and all three sets $A(f), L(f)$, and $Z(f)$ are completely invariant;

3. $J(f)=\partial A(f)=\partial L(f)=\partial Z(f)$;

4. every Fatou component $U$ has either $U \cap Z(f)=\emptyset$ or $U \subset Z(f)$; moreover, it has either $U \cap A(f)=\emptyset$ or $\bar{U} \subset A(f)$, and also either $U \cap L(f)=\emptyset$ or $U \subset L(f)$.

5. If $f$ has no wandering domains, then $J(f)=\overline{A(f)}=\overline{Z(f)}$;

6. every (periodic) Baker domain lies in $L(f)$;

7. if $U$ is a Baker wandering domain, then $\bar{U} \subset A(f)$; 
8. if $f$ has a Baker wandering domain, then $A(f)$ and $I(f)$ are connected, and each component of $A(f) \cap J(f)$ is bounded; otherwise, each component of $A(f) \cap$ $J(f)$ is unbounded;

9. every component of $A(f)$ is unbounded;

10. wandering domains may escape slow enough for $L(f)$ and fast enough for $A(f)$.

Item (1) comes out of Eremenko's proof that $I(f)$ is non-empty (compare Theorem 1.8). In (2), invariance is built into the definition, and the inclusion comes from [BH99]. For Statement (3), note that $\partial A(f), \partial L(f)$, and $\partial Z(f)$ are all closed invariant sets, so they contain $J(f)$ by Theorem 1.7, and the claim reduces to the fact that any Fatou component that intersects $A(f), L(f)$, or $Z(f)$ is contained in these sets. This follows from Lemma 4.5 and also implies (4) (see [RS00]). Statement (5) is from [RS00, BH99]. Statement (6) is due to Baker (see Lemma 4.5). Statements (7) and (9) are from [RS05a], while (8) is unpublished recent work by Rippon and Stallard. Wandering domains in $L(f)$ were given in Examples 2.9 and 2.10, while Baker wandering domains are in $A(f)$; this is (10).

Recent work by Rippon and Stallard is on "spider's webs": these are subsets of $I(f)$ for which every complementary component is connected (such as the orbit of a Baker wandering domain, together with certain parts of $I(f)$ connecting the various wandering domains). Rippon and Stallard propose the idea that this feature may be no less common and prototypical for the dynamics of entire functions as Cantor Bouquets are nowadays often considered to be.

\section{Theorem 4.8 (Slow Escape Possible).}

For every real sequence $K_{n} \rightarrow \infty$, there is a $z \in I(f) \cap J(f)$ so that $\left|f^{\circ n}(z)\right|<K_{n}$ for all sufficiently large $n$.

This result is also due to Rippon and Stallard [RS].

It had been observed by Fatou that $I(f)$ often contains curves to $\infty$. This was shown for exponential maps in [DGH], and for more general entire functions having logarithmic tracts satisfying certain geometric conditions in [DT86]. This leads to the following.

\section{Definition 4.9 (Dynamic Ray).}

A dynamic ray tail is an injective curve $\gamma:(\tau, \infty) \rightarrow I(f)$ so that

- $f^{\circ n}(\gamma(t)) \rightarrow \infty$ as $t \rightarrow \infty$ for every $n \geq 0$, and

- $f^{\circ n}(\gamma(t)) \rightarrow \infty$ as $n \rightarrow \infty$ uniformly in $t$.

A dynamic ray is a maximal injective curve $\gamma:(0, \infty) \rightarrow I(f)$ so that for every $\tau>0$, the restriction $\left.\gamma\right|_{(\tau, \infty)}$ is a dynamic ray tail. An endpoint of a dynamic ray $\gamma$ is a point $a$ with $a=\lim _{t \backslash \tau} \gamma(t)$.

Remark 4.10. Dynamic rays are sometimes called "hairs"; we prefer the term "ray" in order to stress the similarity to the polynomial case.

Eremenko asked whether every point in $I(f)$ can be connected to $\infty$ by a curve in $I(f)$ (compare Conjecture 4.2). This conjecture has been confirmed for exponential 
maps with attracting fixed points by Devaney and Krych [DK84] (in this case, the Julia set is an uncountable collection of curves), in [SZ03a] for arbitrary exponential maps (including the case when $J(f)=\mathbb{C}$ ), and in [RoS08] for arbitrary cosine maps $z \mapsto a e^{z}+b e^{-z}$. For functions of bounded type and finite order, this was shown by Barański [Bar07] under the additional condition that there is an attracting fixed point that attracts all singular values, and by Rottenfußer, Rückert, Rempe and Schleicher [RRRS] in general. We state a particular case of the result here (the precise statement in [RRRS] is phrased in geometric terms on the tracts).

\section{Theorem 4.11 (Dynamic Rays, Bounded Type and Finite Order).}

For entire functions of bounded type and finite order, or compositions thereof, $I(f)$ consists entirely of rays, possibly with endpoints (the strong version of Eremenko's conjecture holds).

However, there are counterexamples to this question of Eremenko, even for entire functions of bounded type; the counterexamples of course have infinite order, but they can be quite close to having finite order [RRRS] (these had been mentioned in Theorem 3.16).

In many cases, points on dynamic rays are in $A(f)$. This is recent unpublished work by Rippon and Stallard. This is the case if $f$ is a composition of functions of finite order and bounded type, and more generally for all functions in [RRRS] that satisfy an additional "bounded gulfs" condition.

Certain Julia sets, especially of hyperbolic entire functions, are described in terms of Cantor Bouquets. Roughly speaking, a Cantor bouquet $X$ is an uncountable union of disjoint curves in the Julia set (dynamic rays with endpoints) so that $X$ has no interior point and each point in $X$ has a neighborhood that intersects $X$ in a set that contains a homeomorphic copy of a Cantor set cross an interval (but every neighborhood of every point in the Cantor bouquet also contains endpoints of curves, so locally $X$ is not homeomorphic to a Cantor set cross an interval). Prototypical examples of Cantor bouquets are Julia sets of exponential maps with attracting fixed points [DK84], but there is a substantial body of work identifying Cantor bouquets in many more Julia sets of entire functions (see, for instance, [DT86] or recent work by Rempe and coauthors). Aarts and Oversteegen [AO93] have given an abstract definition of Cantor bouquets, and they have shown some universality properties.

We conclude this section with an amusing result by Mayer [Ma90].

\section{Definition 4.12 (Explosion Set).}

A set $X \subset \mathbb{C}$ is called an explosion set if $X \cup\{\infty\}$ is connected in $\overline{\mathbb{C}}$, but $X$ is totally disconnected.

\section{Theorem 4.13 (Explosion Set).}

For every exponential map $z \mapsto \lambda e^{z}$ with an attracting fixed point, the landing points of dynamic rays form an explosion set in $\mathbb{C}$.

It seems likely that an analogous result should hold in much greater generality, possibly for bounded type entire functions of finite order, at least when there is an attracting fixed point attracting all singular values. 


\section{Hausdorff Dimension}

In this section, we describe a number of results on the Hausdorff dimension of Julia sets of entire functions. We will start with a few results on specific prototypical families and conclude the section with a collection of results on more general maps. The first two results are due to McMullen [Mc87].

\section{Theorem 5.1 (Hausdorff Dimension 2).}

For every map $z \mapsto \lambda e^{z}$, the Julia set (and even the escaping set) has Hausdorff dimension 2.

\section{Theorem 5.2 (Julia Set of Positive Measure).}

For every map $z \mapsto a e^{z}+b e^{-z}$ with $a b \neq 0$, the Julia set (and even the escaping set) has positive 2-dimensional Lebesgue measure.

In this section, we will only sketch some of the proofs (see also the review article [Sch07a]); they use parabolas of the following type (for $\xi>0$ and $p>1$ ):

$$
R_{p, \xi}:=\left\{x+i y \in \mathbb{C}:|x|>\xi \text { and }|y|<|x|^{p}\right\} .
$$

For large $p$, the set $R_{p, \xi}$ is the complement to a narrow parabola around the imaginary axis.

The proof of Theorem 5.2 goes roughly as follows: we consider a "standard square" $Q \subset R_{p, \xi}$ with sides parallel to the coordinate axes and of side length $2 \pi$, where $\xi$ is large depending on $a$ and $b$. On $R_{p, \xi}$, the map $E(z)=a e^{z}+b e^{-z}$ "essentially" equals $a e^{z}$ if $\operatorname{Re} z>\xi$, and it equals $b e^{-z}$ if $\operatorname{Re} z<-\xi$. Thus if the real parts of $Q$ are in $[x, x+2 \pi]$, then the image of $Q$ under $E$ almost equals an annulus between radii $e^{x}$ and $e^{2 \pi} e^{x}$ (up to factors of $|a|$ or $\mid b$ ), so the fraction (in terms of Lebesgue area) of $E(Q)$ that falls outside of $R_{p, \xi}$ is on the order of $1 / \sqrt[p]{e^{x}}=e^{-x / p}$; the remaining part has real parts of absolute value at least $e^{x / p}$. The image annulus is covered by a large number of standard squares at much bigger real parts, and the argument can be repeated. The idea is that in every step, the relative fraction of area lost into $\mathbb{C} \backslash R_{p, \xi}$ is so small that the total relative loss, in the product over infinitely many steps, is less than one. In other words, a positive fraction of the area within each standard square is in $I(f)$, so $I(f)$ has positive (in fact, infinite) 2-dimensional Lebesgue measure.

The proof of Theorem 5.1 is similar. We consider the truncated quadrant $S_{\xi}:=$ $\{z \in \mathbb{C}:|\operatorname{Im} z|<\operatorname{Re} z, \operatorname{Re} z>0\}$, and the set of orbits that escape within $S_{\xi}$ : that is, $I_{\xi}:=\left\{z \in I(f):\right.$ the orbit of $z$ is in $\left.S_{\xi}\right\}$. In each step, three quarters of orbits fall outside of $S_{\xi}$, so $I_{\xi}$ has measure zero. We want to estimate its Hausdorff dimension. In this case, a standard square is a square in $S_{\xi}$ of side length $\pi / 2$, again with sides parallel to the coordinate axes, and adjusted vertically so that its images under $E_{\lambda}(z)=\lambda e^{z}$ (which are always one quarter of an annulus) are contained again in $S_{\xi}$. If $Q$ is a standard square, and $E_{\lambda}(Q)$ has real parts greater than $N$, then $E_{\lambda}(Q)$ intersects approximately $N$ horizontal lines of standard squares, each containing $N$ standard squares (up to bounded factors; of course, the number of standard squares 
in each line is different). Transporting the standard squares in $E_{\lambda}(Q)$ back into $Q$, we replace each standard square by approximately $N^{2}$ smaller squares of side length $1 / N$. Again, this process is iterated (which increases $N$ and thus makes the bounded factors irrelevant), and this establishes Theorem 5.1.

The next statement combines results of Karpińska, Schleicher, and Barański [Ka99, SZ03a,Bar07]

\section{Theorem 5.3 (Dynamic Rays of Dimension One).}

For every exponential or cosine map, the set of dynamic rays has Hausdorff dimension 1. The same holds for significantly larger classes of maps, including those of bounded type and finite order which have an attracting fixed point that contains all singular values in its immediate basin.

The leads to the following interesting result discovered by Karpínska: for exponential maps with attracting fixed points, the Julia set is an uncountable union of components, each of which is a dynamic ray with a single endpoint. The dynamic rays have Hausdorff dimension 1 by Theorem 5.3, while the entire Julia set has Hausdorff dimension 2 by Theorem 5.1: therefore, all the dimension sits in the endpoints of the dynamic rays. The situation is even more extreme for cosine maps, where the Julia set has positive Lebesgue measure (Theorem 5.2). For postcritically finite cosine maps, the endpoints of rays are everything but the 1-dimensional set of rays, and we have the following [Sch07b]:

\section{Theorem 5.4 (The Dimension Paradox for Cosine Maps).}

Every postcritically finite cosine map $C(z)=a e^{z}+b e^{-z}$ has the following properties:

- the Julia set equals $\mathbb{C}$;

- every dynamic ray lands at a unique point in $\mathbb{C}$;

- every point in $\mathbb{C}$ is either on a dynamic ray, or it is the landing point of one, two, or four dynamic rays;

- the set of dynamic rays has Hausdorff dimension 1;

- the landing points of these rays are the complement of the one-dimensional set of rays.

The motor for all the results that the Hausdorff dimension of dynamic rays is 1 is the following lemma of Karpińska [Ka99].

\section{Lemma 5.5 (The Parabola Lemma).}

For every map $z \mapsto \lambda e^{z}$ or $z \mapsto a e^{z}+b e^{-z}$, the set of escaping parameters that escape within the parabola $P_{p}=\left\{z \in \mathbb{C}:|\operatorname{Im} z|<|\operatorname{Re} z|^{1 / q}\right\}$ has Hausdorff dimension at most $1+1 / q$.

The idea of this lemma is that points within this parabola are forced to have fast growing real parts, and standard squares as above are replaced by $N^{1+1 / q}$ smaller standard squares (similarly as in the proof of Theorem 5.1, except that the quadrant $\tilde{P}_{\xi}$ is replaced by the parabola $P_{q}$ that occupies ever smaller fractions of large 
centered annuli, as the radii increase). For details, see [Ka99,Sch07a]. The topological claims in Theorem 5.4 use standard contraction arguments using the hyperbolic metric in the finite postsingular set; see [Sch07b].

The results cited so far show that escaping points of exponential maps have the property that all the points on the dynamic rays have dimension 1 , while the endpoints have dimension 2. A more precise study, investigating how the endpoints can be subdivided into sets of dimensions between 1 and 2, was done by Karpiǹska and Urbański [KU06].

We already mentioned in Section 4 that Rippon and Stallard have new results showing that in much greater generality, points on dynamic rays escape to $\infty$ as fast as possible; this should imply that in many cases, the dimension of dynamic rays equals 1. Barański [Bar08] has shown this for bounded type and finite order maps for which a single attracting fixed point absorbs all singular orbits, and results by Rempe [Re] allow to lift the condition on the attracting fixed point.

The following result on the area of the Fatou set was a conjecture of Milnor and has been shown by Schubert [Schu08]. The proof is in a similar spirit as some of the previous arguments.

\section{Theorem 5.6 (Unbounded Fatou Component of Finite Area).}

For the map $z \mapsto \sin z$, the Fatou set is contained in basins of parabolic fixed points. The Fatou set intersects every vertical strip of width $2 \pi$ in an unbounded set of finite planar Lebesgue measure.

Next we state a useful result of Bock [Bo96].

Theorem 5.7 (Typical Orbits of Entire Functions).

Let $f$ be a non-constant entire function, let $S(f)$ be its set of singular values, and let $P(f):=\overline{\bigcup_{n \geq 0} f^{\circ n}\left(S_{f}\right)}$ be the postsingular set. Then at least one of the following is true:

1. almost every orbit converges to $P(f) \cup\{\infty\}$;

2. $J(f)=\mathbb{C}$ and almost every $z \in \mathbb{C}$ is recurrent.

This result allows to describe more crisply the behavior of typical orbits. A typical consequence is the following: if an entire function has finitely many singular values, and all of them eventually land on repelling cycles, then if $I(f)$ has positive measure it must have full measure in $\mathbb{C}$ (i.e., $\mathbb{C} \backslash I(f)$ must have measure zero). This applies for instance to postcritically finite maps in the cosine family.

Now we state a number of results on more general entire functions. We start by a result of Stallard [St96].

\section{Theorem 5.8 (Hausdorff Dimension Greater than 1).}

For every entire map of bounded type, the Hausdorff dimension of $J(f)$ strictly exceeds 1 .

It is still an open question whether there exists an entire function the Julia set of which has Hausdorff dimension 1. A recent result by Bergweiler, Karpińska, and Stallard is the following [BKS]. 


\section{Theorem 5.9 (Hausdorff Dimension 2).}

For every entire map of bounded type and finite order, the Julia set has Hausdorff dimension 2. More generally, if $f$ has bounded type and for every $\varepsilon>0$ there is a $r_{\varepsilon}>0$ so that

$$
\log \log |f(z)| \leq(\log |z|)^{q+\varepsilon}
$$

for $|z|>r_{\varepsilon}$, then the Julia set has Hausdorff dimension at least $1+1 / q$.

(Note that functions of finite order satisfy the condition for $q=1$, so the second statement generalizes the first.)

The following result is due to Stallard [St97].

\section{Theorem 5.10 (Explicit Values of Hausdorff Dimension).}

For every $p \in(1,2]$, there is an explicit example of an entire function for which the Julia set has Hausdorff dimension p.

Finally we would like to mention the recent survey article on Hausdorff dimension of entire functions by Stallard [St08].

\section{Parameter Spaces}

In addition to the study of individual complex dynamical systems, a substantial amount of attention is given to spaces (or families) of maps. This work comes in at least two flavors: in most of the early work, specific usually complex onedimensional families of maps are considered; in transcendental dynamics, this is most often the family of exponential maps (parametrized as $z \mapsto \lambda e^{z}$ with $\lambda \in \mathbb{D}^{*}$ or $z \mapsto e^{z}+c$ with $c \in \mathbb{C}$ ). Pioneering work in this direction was by Baker and Rippon [BR84], Devaney, Goldberg, and Hubbard [DGH], and by Eremenko and Lyubich [EL92]. Another flavor is to consider larger "natural" parameter spaces. In rational dynamics, such natural parameter spaces are finite-dimensional and easy to describe explicitly (such as the family of polynomials or rational maps of given degree $d \geq 2$ ). In transcendental dynamics, reasonable notions of natural parameter spaces are less obvious. Early work in this direction was done for instance by Eremenko and Lyubich [EL92].

We start with a few remarks on general parameter spaces of entire functions and especially a recent theorem by Rempe. We then discuss the family of exponential maps as the space of prototypical entire functions and compare it with the Mandelbrot set as the space of prototypical polynomials. We conclude this section with a question of Euler.

The following definition is often seen as the natural parameter space of transcendental entire functions of bounded type.

\section{Definition 6.1 (Quasiconformally Equivalent Entire Functions).}

Two functions $f, g$ of bounded type are called quasiconformally equivalent near $\infty$ if there are quasiconformal homeomorphisms $\varphi, \psi: \mathbb{C} \rightarrow \mathbb{C}$ such that $\varphi \circ f=g \circ \psi$ near $\infty$. 
One way to interpret this definition is as follows. We write $g=\left(\varphi \circ f \circ \varphi^{-1}\right) \circ$ $\left(\varphi \circ \psi^{-1}\right)$, so $g$ is a quasiconformally conjugate function to $f$, postcomposed with another quasiconformal homeomorphism. In analogy, every quadratic polynomial is conjugate to one of the form $z^{2}+c$, so any two quadratic polynomial differ from each other by conjugation, postcomposed with an automorphism of $\mathbb{C}$ (here, there are few enough marked points so that for the postcomposition, it suffices to use complex automorphisms).

Theorem 6.2. Let $f, g$ be two entire functions of bounded type that are quasiconformally equivalent near $\infty$. Then there exist $R>0$ and a quasiconformal homeomorphism $\vartheta: \mathbb{C} \rightarrow \mathbb{C}$ so that $\vartheta \circ f=g \circ \vartheta$ on

$$
A_{R}:=\left\{z \in \mathbb{C}:\left|f^{\circ n}(z)\right|>R \text { for all } n \geq 1\right\} .
$$

Furthermore $\vartheta$ has zero dilatation on $\left\{z \in A_{R}:\left|f^{\circ n}(z)\right| \rightarrow \infty\right\}$.

In particular, quasiconformally equivalent entire functions of bounded type are quasiconformally conjugate on their escaping sets. This result may be viewed as an analog to Schröder's theorem (that any two polynomials of equal degree are conformally conjugate in a neighborhood of $\infty$ ); it is due to Rempe [Re], together with the following corollary.

\section{Corollary 6.3 (No Invariant Line Fields).}

Entire functions of bounded type do not support measurable invariant line fields on their sets of escaping points.

A lot of work has been done on the space of the simplest entire functions: that is the space of exponential functions. It plays an important role as a prototypical example, in a similar way as quadratic polynomials play an important prototypical role for polynomials. By now, there is a good body of results on the parameter space of exponential functions, in analogy to the Mandelbrot set. Some of the results go as follows:

\section{Theorem 6.4 (Exponential Parameter Space).}

The parameter space of exponential maps $z \mapsto \lambda e^{z}$ has the following properties:

1. there is a unique hyperbolic component $W$ of period 1 ; it is conformally parametrized by a conformal isomorphism $\mu: \mathbb{D}^{*} \rightarrow W, \mu \mapsto \mu \exp (-\mu)$, so that the map $E_{\lambda}$ with $\lambda=\mu \exp (-\mu)$ has an attracting fixed point with multiplier $\mu$;

2. for every period $n \geq 2$, there are countably many hyperbolic components of period $n$; on each component, the multiplier map $\mu: W \rightarrow \mathbb{D}^{*}$ is a universal covering;

3. for every hyperbolic component $W$ of period $\geq 2$, there is a preferred conformal isomorphism $\Phi: W \rightarrow \mathbb{H}^{-}$with $\mu=\exp \circ \Phi$ (where $\mathbb{H}^{-}$is the left half plane);

4. there is an explicit canonical classification of hyperbolic components and hyperbolic parameters; 
5. the preferred conformal isomorphism $\Phi: W \rightarrow \mathbb{H}^{-}$extends as a homeomorphism $\Phi: \bar{W} \rightarrow \overline{\mathbb{H}}^{-}$; in particular, every hyperbolic component has connected boundary;

6. there is an explicit canonical classification of boundary points of hyperbolic components and of exponential maps with indifferent orbits;

7. escaping parameters (those for which the singular value escapes to $\infty$ ) are organized in the form of parameter rays, together with landing points of certain parameter rays; this yields an explicit classification of all escaping parameters;

8. the Hausdorff dimension of the parameter rays is 1, while the Hausdorff dimension of all escaping parameters (parameter rays and some of their landing points) is 2;

9. exponential parameter space fails to be locally connected at any point on a parameter ray;

10. there is an explicit classification of all parameters for which the singular orbit is finite (i.e., strictly preperiodic);

11. the exponential bifurcation locus is connected.

We will comment on these results, give references, and relate them to results on the Mandelbrot set.

(1), (2), (3), (4) The existence of hyperbolic components of all periods has been shown in several early papers on exponential parameter space [DGH, BR84, EL84b, EL92]; the fact that the multiplier map is a conformal isomorphism onto $\mathbb{D}^{*}$ for period 1 and a universal cover over $\mathbb{D}^{*}$ for period 2 and greater is shown there as well. This shows the existence of a conformal isomorphism $\Phi: W \rightarrow \mathbb{H}^{-}$ with $\mu=\exp \circ \Phi$; it is made unique in [Sch00]. Hyperbolic components are given combinatorial labels in [DFJ02], and they are completely classified in [Sch00]; the latter result leads to a complete classification of exponential maps with attracting periodic orbits. The fact that the component of period 1 is special is related to the parametrization $z \mapsto \lambda e^{z}$ : for instance, for the parametrization $z \mapsto e^{z}+c$, there is still a single hyperbolic component of period 1, but its multiplier map is a universal cover over $\mathbb{D}^{*}$. All these results are in close analogy to the Mandelbrot set and its degree $d$ cousins, the Multibrot sets: these have finitely many hyperbolic components of each period (with an explicit classification), and the multiplier maps on these have degree $d-1$.

(5), (6) The multiplier map of a hyperbolic component clearly extends continuously to the boundary; it was conjectured in the 1980's (by Eremenko and Lyubich [EL84b] and by Baker and Rippon [BR84]) that every component has connected boundary, or equivalently that $\Phi$ extends as a homeomorphism $\Phi: \bar{W} \rightarrow \overline{\mathbb{H}}^{-}$. This conjecture has been confirmed in [Sch04a, RSch09], and this leads to a classification of exponential maps with indifferent orbits. The problem is the following: an internal parameter ray of a hyperbolic component is the $\Phi^{-1}$-image of a horizontal line $\gamma_{y}:=\left\{x+i y \in \mathbb{H}^{-}: x \in(-\infty, 0)\right\} \subset \mathbb{H}^{-}$. It is easy to see that this parameter ray lands at some $\lambda_{y} \in \overline{\mathbb{C}}$, i.e., the limit as $x \rightarrow 0$ of $\Phi^{-1}\left(\gamma_{y}(x)\right)$ exists. The difficulty is to exclude that the limit $\lambda_{y}=\infty$ : if $\lambda_{y} \in \mathbb{C}$, then the exponential map $E_{\lambda_{y}}$ has an 
indifferent orbit with multiplier $e^{i y}$; if not, then the corresponding parameter would be missing from the classification.

(7) Parameter rays in the space of exponential maps are constructed and classified in [FS09]. There are additional parameters for which the singular value escapes; these are landing points of certain parameter rays and are classified in [FRS08].

(8) It is shown in [BBS08] that parameter rays in exponential parameter space have Hausdorff dimension 1, and in [Qiu94] that all escaping parameters have Hausdorff dimension 2. By [FRS08], every escaping parameter is either on a parameter ray or a landing point of one of them.

(9) Contrary to one of the principal conjectures on the Mandelbrot set, and many combinatorial similarities between the parameter spaces of exponential maps and quadratic polynomials, the exponential bifurcation locus is not locally connected at any point on any parameter ray: in fact, any parameter ray is approximated by other parameter rays on both sides, and between any pair of parameter rays there are are hyperbolic components. This destroys local connectivity of the exponential bifurcation locus [RSch08].

(10) For rational maps, there is a fundamental theorem of Thurston [DH93] that characterizes rational maps with finite critical orbit and that is at the basis of most classification theorems in rational dynamics. Unfortunately, there is no analogous result for transcendental maps. Currently, the only extension of Thurston's theorem to the case of transcendental maps is [HSS09] on exponential maps with finite singular orbits (see also [Se09] for work in progress in this direction); the resulting classification of postsingularly finite exponential maps is in [LSV08]. This classification had been expected for a long time [DGH].

(11) The fundamental study of the Mandelbrot starts with Douady and Hubbard's result about connectivity of the Mandelbrot set, or equivalently of its boundary, which is the bifurcation locus in the space of quadratic polynomials. The corresponding result about exponential maps is that the exponential bifurcation locus is connected. Unlike in the polynomial case, where connectivity of the bifurcation locus of quadratic polynomials is the starting point for much of the theory of the Mandelbrot set, this result comes at the end of a detailed study of exponential parameter space. It was shown in [RSch09].

Just like for the whole field of entire dynamics, it is impossible to do justice to the large body of knowledge that has been established from many different points of view even on exponential parameter space. Among further existing work, we would like to mention [GKS04,UZ07, Ye94]. There is also a significant amount of work on other one-dimensional parameter spaces of explicit entire functions; we only mention the work by Fagella, partly with coauthors, on the families $z \mapsto \lambda z e^{z}$ [Fa99] and on $z \mapsto \lambda z^{m} e^{z}$ (with $m \geq 2$ ) [FaGa07]. Even though these are not entire functions, we would also like to mention work on the family of tangent maps by [KK97].

\section{Conjecture 6.5 (Exponential Parameter Space).}

- Hyperbolicity is dense in the space of exponential maps.

- Fibers in exponential parameter space are trivial. 
Remark 6.6. Fibers in exponential parameter space are defined in analogy as for the Mandelbrot set (see [RSch08] for the exponential case and [Sch04b] for the Mandelbrot case). The second conjecture says that all non-hyperbolic exponential maps are combinatorially rigid (their landing patterns of periodic dynamic rays differ); it is the analog to the famous conjecture that the Mandelbrot set is locally connected. The second conjecture implies the first [RSch08].

We conclude this section with a question of Euler [E1777] that was raised in 1777, and its generalization to complex numbers.

\section{Question 6.7 (Euler Question: Iterated Exponentiation).}

For which real $a>0$ does the sequence $a, a^{a}, a^{a^{a}}, a^{a^{a^{a}}}, \ldots$, have a limit?

This question can be rephrased, by setting $\lambda:=\log a$, as follows: for which $\lambda \in \mathbb{R}$ does the sequence $x_{0}:=0, x_{n+1}=\lambda e^{x_{n}}$, have a limit? In this form, it makes sense for $\lambda \in \mathbb{C}^{*}$. The answer is surprisingly complicated. Set $E_{\lambda}(z):=\lambda e^{z}$. To begin with, we note that if the sequence $x_{n}$ converges to some $b \in \mathbb{C}$ but is not eventually constant, then $b$ must be an attracting or parabolic fixed point (clearly, no repelling fixed point and no center of a Siegel disk can be a limit point of an orbit unless the latter is eventually constant; and it is not hard to show that a Cremer point cannot be the limit of the unique singular orbit).

The map $E_{\lambda}$ has an attracting fixed point if and only if $\lambda=\mu e^{-\mu}$ with $\mu \in \mathbb{D}^{*}$ (since 0 is the only singular value of $E_{\lambda}$, it follows that in these cases, the orbit of 0 must converge to the attracting fixed point, by Theorem 2.3). Similarly, $E_{\lambda}$ has a rationally indifferent fixed point if and only if $\lambda=\mu e^{-\mu}$ and $\mu$ is a root of unity. This takes care of all cases where the orbit of 0 converges to a finite limit point in $\mathbb{C}$ without eventually being constant. (The analogous question for periodic limit points leads to the classification of hyperbolic components and their boundaries in exponential parameter space, and thus items (4) and (6) in Theorem 6.4.)

The description of parameters $\lambda$ in which the orbit of 0 is eventually fixed (or eventually periodic) involves a classification of postsingularly finite exponential maps, and this is settled by item (10) in Theorem 6.4. Finally, the case of parameters $\lambda$ in which the orbit of 0 converges to $\infty$ is item (7) in that theorem.

\section{Newton Maps of Entire Functions}

If $f$ is an entire holomorphic function, then its associated Newton map $N_{f}:=$ id $-f / f^{\prime}$ is a meromorphic function that naturally "wants to be" iterated. While the iteration of general meromorphic functions falls outside of the scope of this manuscript, there are a number of results specifically on Newton maps of entire functions. In rational iteration theory, polynomials are the easiest maps to work on, and their Newton maps have useful properties that make them easier to investigate than general rational maps. Since we believe that the situation should be similar for in the transcendental world, this section is included. 


\section{Definition 7.1 (Basin and Immediate Basin).}

For a root $\alpha$ of $f$, we define its basin as $U_{\alpha}:=\left\{z \in \mathbb{C}: N_{f}^{\circ n}(z) \rightarrow \alpha\right\}$ as $n \rightarrow \infty$. The immediate basin is the connected component of $U_{\alpha}$ containing $\alpha$.

\section{Theorem 7.2 (Immediate Basins Simply Connected).}

Every root of $f$ has simply connected immediate basin.

This was shown in [MS06]. It is an open question whether every Fatou component of $N_{f}$ is simply connected. After work by Taixes, the last open case is whether Baker domains are always simply connected (compare [FJT08]).

\section{Theorem 7.3 (Wandering Newton Domains Simply Connected).}

If a Newton map has a wandering domain, then it is simply connected.

This follows from work of Bergweiler and Terglane [BT96]: in analogy with classical work of Shishikura [Sh09], they prove that a multiply connected wandering domain of a transcendental meromorphic map $g$ (such as a Newton map) would require that $g$ has a weakly repelling fixed point; but this is not the case for Newton maps.

\section{Definition 7.4 (Virtual Immediate Basin).}

A virtual immediate basin is a maximal subset of $\mathbb{C}$ (with respect to inclusion) among all connected open subsets of $\mathbb{C}$ in which the dynamics converges to $\infty$ locally uniformly and which have an absorbing set. (An absorbing set in a domain $V$ is a subset $A$ such that $N_{f}(\bar{A}) \subset A$ and every compact $K \subset V$ has a $n \geq 0$ so that $N_{f}^{\circ n}(K) \subset A$.)

\section{Theorem 7.5 (Virtual Immediate Basins Simply Connected).}

Every virtual immediate basin is simply connected.

This was also shown in [MS06]. Every virtual immediate basin is contained in a Baker domain; it is an open question whether this basin equals a Baker domain (this is true for simply connected Baker domains). The principal difficulty is the question whether every Baker domain has an absorbing set as in Definition 7.4; this would also imply that every Fatou component of a Newton map is simply connected.

\section{Theorem 7.6 (Two Accesses Enclose Basin).}

Let $f$ be an entire function (polynomial or transcendental) and let $U_{\alpha}$ be the immediate basin of $\alpha$ for $N_{\alpha}$. Let $\Gamma_{1}, \Gamma_{2} \subset U_{\alpha}$ represent two curves representing different invariant accesses to $\infty$, and let $W$ be an unbounded component of $\mathbb{C} \backslash\left(\Gamma_{1} \cup \Gamma_{2}\right)$. Then $W$ contains the immediate basin of a root of $f$ or a virtual immediate basin, provided the following finiteness condition holds: $N_{f}^{-1}(z) \cap W$ is finite for all $z \in \mathbb{C}$.

Remark 7.7. In the case of a polynomial $f$, the finiteness condition always holds, and there is no virtual immediate basin. The result thus says that any two accesses of any immediate basins enclose another immediate basin. Theorem 7.6 is due to Rückert and Schleicher [RüS07]. 
For polynomials, it is known that any two invariant accesses to $\infty$ within the basin of any root always enclose some other root. The analogous statement is false for entire functions: if $f(z)=z e^{e^{z}}$, then the Newton map is $N_{f}(z)=z\left(1-1 /\left(1+e^{z}\right)\right)$. The map $f$ has a single root with infinitely many invariant accesses, but these cannot surround any further root: they indeed surround virtual basins. This example is due to S. Mayer [May] (see also [MS06]).

Virtual immediate basins may be thought of as basins of a root at $\infty$. The prototypical case is $f(z)=\exp (z), N_{f}(z)=z-1$ : there is no root, all points converge to $-\infty$ under $N_{f}$, and indeed $f$ converges to 0 along these orbits. Douady thus asked whether there was a relation between asymptotic values 0 of $f$ and virtual immediate basins. This is indeed often the case. The following two results are due to Buff and Rückert [BR06].

\section{Theorem 7.8 (Logarithmic Singularity Implies Virtual Immediate Basin).}

If $f$ has an asymptotic value 0 which is a logarithmic singularity, then $N_{f}$ has a virtual immediate basin.

There is a partial converse as follows.

\section{Theorem 7.9 (Virtual Immediate Basin and Asymptotic Value).}

Let $V$ be a virtual immediate basin with absorbing set $A$. If the quotient $A / N_{f}$ has sufficiently large modulus, then $f$ has an asymptotic value 0 .

Remark 7.10. There are indeed counterexamples when $A / N_{f}$ has small modulus [BDL07].

\section{Theorem 7.11 (Rational Newton Map).}

The Newton map $N_{f}$ of $f$ is rational if and only if $f=p e^{q}$ for polynomials $p$ and $q$. In this case, $\infty$ is a parabolic fixed point with multiplier 1 and multiplicity $\operatorname{deg}(q)+1$.

This is probably a folklore result; for a proof, which is not difficult, see [RüS07]. Another result that is folklore (at least in the polynomial case) is the following (see again [RüS07]).

\section{Theorem 7.12 (Characterization of Newton Maps).}

A meromorphic function $g$ is the Newton map of an entire function if and only if all fixed points $\xi$ of $g$ satisfy $g^{\prime}(\xi)=(m-1) / m$ for a positive integer $m$. Two entire functions $f_{1}$ and $f_{2}$ have identical Newton maps if and only if $f_{1} / f_{2}$ is constant.

The next result is due to Haruta [Ha99].

\section{Theorem 7.13 (Area of Immediate Basins).}

For $f=p e^{q}$ with polynomials $p$ and $q$ and $\operatorname{deg} q \geq 3$, every immediate basin has finite Lebesgue area in the plane.

We conclude with the following conjecture from [Sch08] on root finding of the Riemann $\zeta$ function by Newton's method. Let $\xi$ be the entire function the roots of which are the non-trivial roots of $\xi$ (so that $\xi(s)=\xi(1-s)$ ). 


\section{Conjecture 7.14 (The Riemann $\zeta$ function).}

There are constants $c, s>0$ with the following property. If there is a root $\alpha$ of $\xi$ whose immediate basin does not contain one of the points $c_{n}^{ \pm}:= \pm 2+c n / \log |n|$, then the Riemann hypothesis is false and there is a root $\alpha^{\prime}$ off the critical line with $\left|\alpha^{\prime}-\alpha\right|<s$ and the immediate basin of $\alpha^{\prime}$ contains a point $c_{n}^{ \pm}$.

Remark 7.15. The preceding conjecture says that the points $c_{n}$ form an efficient set of starting points for finding all roots of $f$, so that the first $N$ starting points find at least $c^{\prime \prime} N$ distinct roots of $\xi$; and if they do not find all roots, then close to a missed root these starting points find a root that violates the Riemann hypothesis.

More results on the dynamics of Newton maps for entire functions can be found in the survey [Rü08].

\section{A Few Open Questions}

There has been an enormous amount of work on the dynamics of transcendental entire (and meromorphic) functions, but many open questions remain (or rather, and therefore, there are many more open questions that await their answers). We just mention a couple of them in order to show that this field continues to be active.

Question 8.1. Is the escaping set $I(f)$ connected for every entire function $f$ ?

Question 8.2. Is there an entire function with a wandering domain with bounded orbit?

Eremenko and Lyubich [EL87] constructed an example of a wandering domain on which the iterates do not tend to $\infty$.

Question 8.3. Is there an entire function of bounded type that has a wandering domain?

(By Theorem 3.4, this entire function could not be of finite type, and the wandering domain could not consist of escaping orbits.)

Question 8.4. If an entire function has order less than $1 / 2$, does this imply that the Fatou set has no unbounded Fatou components?

This is a conjecture by Baker, and it has inspired a lot of work and partial results, such as by Baker, Stallard, Anderson-Hinkkanen, and others. It is still open even for functions of order 0 . Another open question is whether $A(f)$, the set of points that escape to infinity as fast as possible, can have unbounded Fatou components.

Question 8.5. Is there an entire function whose Julia set has Hausdorff dimension 1 ? 
As discussed in Section 5, the Hausdorff dimension of the Julia set always has dimension at least 1 , and there are examples of entire functions for which the dimension of the Julia set is arbitrarily close to 1 . For functions of bounded type, the Julia set always has Hausdorff dimension strictly greater than 1 .

Question 8.6. Does every Newton map of an entire function always have connected Julia set?

This is true for polynomials, and there are positive partial results in the transcendental case. The main difficulty seems to be with Baker domains. (See Section 7.)

We have not discussed irrationally indifferent periodic points of entire functions. The following is an open question even for polynomials of arbitrary degree, but there are complete answers for certain spaces of maps (such as quadratic polynomials, by Yoccoz' linearization condition[Y95], and maps $z \mapsto \lambda z e^{z}$, by Geyer [Ge01]).

Question 8.7. Give, for various classes of entire functions, a necessary and sufficient condition for an irrationally indifferent periodic point to have a Siegel disk.

We now state a couple of questions that are more specific for the family of exponential maps; but many of these questions can also be asked for larger classes of entire functions: the greater question is "investigate, for various known properties of exponential maps, under which conditions these hold for larger classes of entire functions".

Question 8.8. Suppose for an exponential map $z \mapsto \lambda \exp (z)$, the singular value does not converge to $\infty$. Is every repelling periodic point the landing point of a periodic dynamic ray? Is there a generalization to larger classes of maps (of bounded type and finite order)?

Question 8.9. Suppose for an exponential map $z \mapsto \lambda e^{z}$, the Julia set equals $\mathbb{C}$. Does this imply that there is a dynamic ray that does not land? Is there an analogous result for entire functions without critical values? Or for those that have finite asymptotic values?

Question 8.10. Characterize the parameters $\lambda \in \mathbb{C}^{*}$ for which the set of nonescaping points in $\mathbb{C}$ of $z \mapsto \lambda e^{z}$ is connected; characterize also those parameters for which the set of non-escaping points union $\{\infty\}$ is connected.

Question 8.11. Show that hyperbolicity is dense in the space of exponential maps.

The analogous question remains open for any non-trivial space of holomorphic mappings, even for the prototypical case of quadratic polynomials.

We conclude this section by a question on a very specific map that would have amusing consequences.

Question 8.12. Show that the following map does not have a wandering domain (or at least not one that intersects the real axis):

$$
f(z)=z / 2+(1-\cos \pi z)(z+1 / 2) / 2+((1 / 2-\cos \pi z) \sin \pi z) / \pi
$$


Remark 8.13. The relevance of the last question is the following: it is known that $\mathbb{Z}$ is in the Fatou set for this function $f$. Moreover, on $\mathbb{Z}$, the function $f$ coincides with the well-known $3 n+1$ problem: $f(n)=n / 2$ if $n$ even and $f(n)=(3 n+1) / 2$ if $n$ is odd. Therefore, solving this question would prove that the $3 n+1$ problem does not have an orbit tending to $\infty$ (it would still be possible for $f$ to have periodic integer orbits other than the cycle $1 \mapsto 2 \mapsto 1$; such as the fixed point -1 or $-5 \mapsto-7 \mapsto$ $-10 \mapsto-5)$. See [LSW99] for more details.

\section{A. Background and Notation}

In this brief appendix, we collect some of the notation that we are using in this text, and we state some of the standard results from complex analysis that we require.

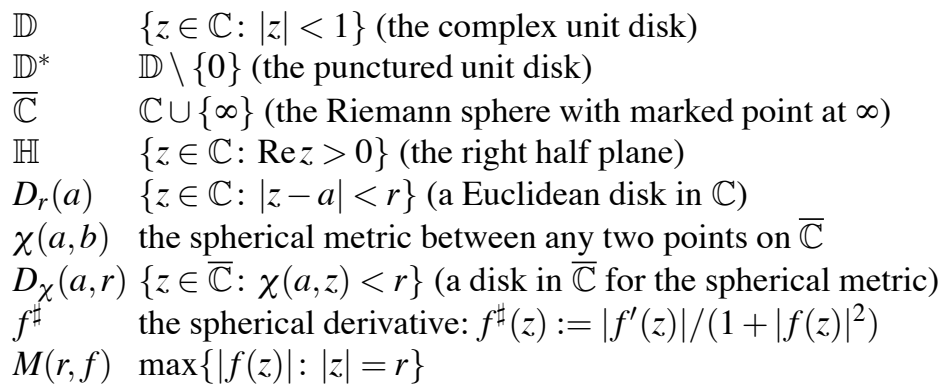

\section{Definition A.1 (Normal Family).}

Let $U \subset \mathbb{C}$ be a domain. A family $\left(f_{k}\right)$ of holomorphic maps $U \rightarrow \overline{\mathbb{C}}$ is called a normal family if every sequence of the $\left(f_{k}\right)$ contains a subsequence that converges locally uniformly to a holomorphic limit function $f: U \rightarrow \overline{\mathbb{C}}$.

Being a normal family is a local property: if every $z \in U$ has a neighborhood on which a family of maps is normal, then the family is normal on all of $U$ (this follows by a standard diagonal argument).

\section{Theorem A.2 (Montel's Theorem).}

Any family $\left(f_{k}\right): U \rightarrow \overline{\mathbb{C}} \backslash\{a, b, c\}$ of holomorphic functions from a domain $U \subset \overline{\mathbb{C}}$ to the sphere with three distinct punctures $a, b, c$ is normal.

\section{Theorem A.3 (Marty's Criterion).}

A family $\left(f_{k}\right): U \rightarrow \overline{\mathbb{C}}$ of holomorphic maps is normal if and only if the spherical derivatives are locally bounded.

\section{Theorem A.4 (Picard's Theorem).}

If $f: \mathbb{D}^{*} \rightarrow \overline{\mathbb{C}}$ has an essential singularity at 0 , then $f$ assumes every $z \in \overline{\mathbb{C}}$, with at most two exceptions, infinitely often in every neighborhood of 0 .

General references to the results in this appendix include [A98,H64,S93] as well as [Mi06, Be98]. 


\section{References}

[AO93] Aarts, J.M., Oversteegen, L.G.: The geometry of Julia sets. Trans. Am. Math. Soc. 338(2), 897-918 (1993)

[Ab] Abate, M.: Discrete holomorphic local dynamical systems, course notes, this volume

[A98] Ahlfors, L.: Complex analysis, 3rd edn. International Series in Pure and Applied Mathematics. McGraw-Hill Book Co., New York (1978)

[BBS08] Bailesteanu, M., Balan, V., Schleicher, D.: Hausdorff dimension of exponential parameter rays and their endpoints. Nonlinearity 21(1), 113-120 (2008)

[Ba60] Baker, I.N.: The existence of fixpoints of entire functions. Math. Zeitschr. 73, 280 284 (1960)

[Ba63] Baker, I.N.: Multiply connected domains of normality in iteration theory. Math. Z. 81, 206-214 (1963)

[Ba76] Baker, I.N.: An entire function which has wandering domains. J. Austral. Math. Soc. Ser. A 22(2), 173-176 (1976)

[Ba81] Baker, I.N.: The iteration of polynomials and transcendental entire functions. J. Austral. Math. Soc. Ser. A 30, 483-495 (1980/81)

[Ba84] Baker, I.N.: Wandering domains in the iteration of entire functions. Proc. Lond. Math. Soc. (3) 49(3), 563-576 (1984)

[BKL91] Baker, I.N., Kotus, J., Lü, Y.N.: Iterates of meromorphic functions. III. Preperiodic domains. Ergod. Theor. Dyn. Syst. 11(4), 603-618 (1991)

[BR84] Baker, I.N., Rippon, P.: Iteration of exponential functions. Ann. Acad. Sci. Fenn. Ser. A I Math. 9, 49-77 (1984)

[Bar07] Barański, K.: Trees and hairs for some hyperbolic entire maps of finite order. Math. Z. 257(1), 33-59 (2007)

[Bar08] Barański, K.: Hausdorff dimension of hairs and ends for entire maps of finite order. Math. Proc. Cambridge Philos. Soc. 145(3), 719-737 (2008)

[Ben] Benini, A.: Combinatorial rigidity for Misiurewicz parameters. Manuscript, in preparation.

[Ba99] Bargmann, D.: Simple proofs of some fundamental properties of the Julia set. Ergod. Theor. Dyn. Syst. 19(3), 553-558 (1999)

[Ba01] Bargmann, D.: Normal families of covering maps. J. Analyse. Math. 85, 291-306 (2001)

[BB01] Bargmann, D., Bergweiler, W.: Periodic points and normal families. Proc. Am. Math. Soc. 129(10), 2881-2888 (2001)

[BD00] Berteloot, F., Duval, J.: Une démonstration directe de la densité des cycles répulsifs dans l'ensemble de Julia. Progr. Math. 188, Birkhäuser, Basel, 221-222 (2000)

[Be91] Bergweiler, W.: Periodic points of entire functions: proof of a conjecture of Baker. Complex Variables Theory Appl. 17, 57-72 (1991)

[Be93] Bergweiler, W.: Iteration of meromorphic functions. Bull. Am. Math. Soc. (N.S.) 29(2), 151-188 (1993)

[Be] Bergweiler, W.: An entire function with simply and multiply connected wandering domains, to appear in Pure Appl. Math. Quarterly

[Be98] Bergweiler, W.: A new proof of the Ahlfors five islands theorem. J. Anal. Math. 76, 337-347 (1998)

[BDL07] Bergweiler, W., Drasin, D., Langley, J.K.: Baker domains for Newton's method. Ann. Inst. Fourier (Grenoble) 57(3), 803-814 (2007)

[BE95] Bergweiler, W., Eremenko, A.: On the singularities of the inverse to a meromorphic function of finite order. Rev. Mat. Iberoamericana 11(2), 355-373 (1995)

[BHKMT93] Bergweiler, W., Haruta, M., Kriete, H., Meier, H., Terglane, N.: On the limit functions of iterates in wandering domains. Ann. Acad. Sci. Fenn. Ser. A I Math. 18(2), 369-375 (1993) 
[BH99] Bergweiler, W., Hinkkanen, A.: On semiconjugation of entire functions. Math. Proc. Cambridge Philos. Soc. 126(3), 565-574 (1999)

[BKS] Bergweiler, W., Karpińska, B., Stallard, G.: The growth rate of an entire function and the Hausdorff dimension of its Julia set. J. Lond. Math. Soc., doi: $10.1112 / \mathrm{jlms} / \mathrm{jdp} 042$

[BT96] Bergweiler, W., Terglane, N.: Weakly repelling fixpoints and the connectivity of wandering domains. Trans. Am. Math. Soc. 348(1), 1-12 (1996)

[BRS08] Bergweiler, W., Rippon, P., Stallard, G.: Dynamics of meromorphic functions with direct or logarithmic singularities. Proc. Lond. Math. Soc. 97, 368-400 (2008)

[Bo96] Bock, H.: On the dynamics of entire functions on the Julia set. Results Math.30(12), 16-20 (1996)

[BS] Bruin, H., Kaffl, A., Schleicher, D.: Symbolic Dynamcis of Quadratic Polynomials. Monograph, in preparation. Earlier version: Mittag-Leffler Preprint 7 $(2001 / 02)$

[BR06] Buff, X., Rückert, J.: Virtual immediate basins of Newton maps and asymptotic values. Int. Math. Res. Not., Art. ID 65498, 18 (2006)

[DGH] Devaney, R., Goldberg, L., Hubbard, J.: A dynamical approximation to the exponential map of polynomials. Preprint, MSRI (1986). See [BDGHHR99].

[BDGHHR99] Bodelon, C., Devaney, R., Goldberg, L., Hayes, M., Hubbard, J., Roberts, G.: Hairs for the complex exponential family. Internat. J. Bif. Chaos 9(8), 1517-1534 (1999)

[DK84] Devaney, R., Krych, M.: Dynamics of $\exp (z)$. Ergod. Theor. Dyn. Syst. 4(1), 35-52 (1984)

[DT86] Devaney, R., Tangerman, F.: Dynamics of entire functions near the essential singularity. Ergod. Theor. Dyn. Syst. 6(4), 489-503 (1986)

[DFJ02] Devaney, R., Fagella, N., Jarque, X.: Hyperbolic components of the complex exponential family. Fund. Math. 174(3), 193-215 (2002)

[Do98] Domínguez, P.: Dynamics of transcendental meromorphic functions. Annales Academiæ Scientiarum Fennicæ, Mathematica 23, 225-250 (1998)

[DH93] Douady, A., Hubbard, J.: A proof of Thurston's topological characterization of rational functions. Acta Math. 171, 263-297 (1993)

[Ep] Epstein, A.: Infinitesimal thurston rigidity and the fatou-shishikura inequality. Stony Brook IMS preprint 1 (1999)

[Er78] Eremenko, A.: The set of asymptotic values of a finite order meromorphic function (Russian). Mat. Zametki 24(6), 779-783, 893 (1978). (English translation: Math. Notes 24(5-6), 914-916 (1978))

[Er89] Eremenko, A.: On the iteration of entire functions. Banach Center Publ. 23, PWN, Warsaw, 339-345 (1989)

[EL84a] Eremenko, A., Lyubich, M.: Iterates of entire functions. Soviet Math. Dokl.30(3), 592-594 (1984)

[EL84b] Eremenko, A., Lyubich, M.: Iterates of entire functions (Russian). Preprint, Institute for Low Temperature, Kharkov 6 (1984)

[EL87] Eremenko, A., Lyubich, M.: Examples of entire functions with pathological dynamics. J. Lond. Math. Soc. 36, 458-468 (1987)

[EL89] Eremenko, A., Lyubich, M.: The dynamics of analytic transformations (Russian). Algebra i Analiz 1(3), 1-70 (1989); translation in Leningrad Math. J. 1(3), 563-634 (1990)

[EL92] Eremenko, A., Lyubich, M.: Dynamical properties of some classes of entire functions. Annales Inst. Fourier 42(4), 989-1020 (1992)

[E1777] Euler, L.: De formulis exponentialibus replicatis. Acta Acad. Petropolitanae, 1, 38-60 (1777)

[Fa99] Fagella, N.: Dynamics of the complex standard family. J. Math. Anal. Appl. 229(1), 1-31 (1999)

[FaGa07] Fagella, N., Garijo, A.: The parameter planes of $\lambda z^{m} \exp (z)$ for $m \geq 2$. Comm. Math. Phys. 273(3), 755-783 (2007) 
[FJT08] Fagella, N., Jarque, X., Taixés, J.: On connectivity of Julia sets of transcendental meromorphic maps and weakly repelling fixed points I. Proc. Lond. Math. Soc. 97(3), 599-622 (2008)

[Fa26] Fatou, P.: Sur l'itération des fonctions transcendantes entières. Acta Math. 47, 337-370 (1926)

[FS09] Förster, M., Schleicher, D.: Parameter rays in the space of exponential maps. Ergod. Theor. Dyn. Syst. 29, 515-544 (2009)

[FRS08] Förster, M., Rempe, L., Schleicher, D.: Classification of escaping exponential maps. Proc. Am. Math. Soc. 136, 651-663 (2008)

[Ge01] Geyer, L.: Siegel discs, Herman rings and the Arnold family. Trans. Am. Math. Soc. 353(9), 3661-3683 (2001)

[GE79] Gol'dberg, A., Eremenko, A.: Asymptotic curves of entire functions of finite order. Mat. Sb. (N.S.) 109(151)(4), 555-581, 647 (1979)

[GK86] Goldberg, L., Keen, L.: A finiteness theorem for a dynamical class of entire functions. Ergod. Theor. Dyn. Syst. 6, 183-192 (1986)

[GKS04] Graczyk, J., Kotus, J., Świátek, G.: Non-recurrent meromorphic functions. Fund. Math. 182(3), 269-281 (2004)

[Gr18a] Gross, W.: Über die Singularitäten analytischer Funktionen. Monatsh. Math. Phys. 29(1), 3-47 (1918)

[Gr18b] Wilhelm, G.: Eine ganze Funktion, für die jede komplexe Zahl Konvergenzwert ist (German). Math. Ann. 79(1-2), 201-208 (1918)

[Ha99] Haruta, M.: Newton's method on the complex exponential function. Trans. Am. Math. Soc. 351(6), 2499-2513 (1999)

[H64] Hayman, W.: Meromorphic Functions. Oxford Mathematical Monographs, Clarendon Press, Oxford (1964)

[He57] Heins, M.: Asymptotic spots of entire and meromorphic functions. Ann. Math. (2) 66, 430-439 (1957)

[HSS09] Hubbard, J., Schleicher, D., Shishikura, M.: Exponential Thurston maps and limits of quadratic differentials. J. Am. Math. Soc. 22, 77-117 (2009)

[Iv14] Iversen, F.: Recherches sur les fonctions inverses des fonctions méromorphes. Thèse, Helsingfors, 1914 (see [Ne53]).

[Ka99] Karpińska, B.: Hausdorff dimension of the hairs without endpoints for $\lambda \exp (z)$. C. R. Acad. Sci. Paris Séer. I Math. 328, 1039-1044 (1999)

[KU06] Karpińska, B., Urbański, M.: How points escape to infinity under exponential maps. J. Lond. Math. Soc. (2) 73(1), 141-156 (2006)

[KK97] Keen, L., Kotus, J.: Dynamics of the family $\lambda \tan z$. Conform. Geom. Dyn. 1, 28-57 (1997)

[KS08] Kisaka, M., Shishikura, M.: On multiply connected wandering domains of entire functions. In: Phil Rippon, Gwyneth Stallard (eds.) Transcendental Dynamics and Complex Analysis, London Math. Soc. Lecture Note Ser. 348, Cambridge University Press, Cambridge (2008)

[LSV08] Laubner, B., Schleicher, D., Vicol, V.: A combinatorial classification of postsingularly preperiodic complex exponential maps. Discr. Cont. Dyn. Syst. 22(3), 663-682 (2008)

[LSW99] Letherman, S., Schleicher, D., Wood, R.: On the 3n+1-problem and holomorphic dynamics. Exp. Math. 8(3), 241-251 (1999)

[Ma90] Mayer, J.: An explosion point for the set of endpoints of the Julia set of $\lambda \exp (z)$. Ergod. Theor. Dyn. Syst. 10(1), 177-183 (1990)

[May] Mayer, S.: Newton's Method for Entire Functions. Diplomarbeit, Technische Universität München (2002)

[MS06] Mayer, S., Schleicher, D.: Immediate and virtual basins of Newton's method for entire functions. Ann. Inst. Fourier (Grenoble) 56(2), 325-336 (2006)

[Mc87] McMullen, C.: Area and Hausdorff dimension of Julia sets of entire functions. Trans. Am. Math. Soc. 300(1), 329-342 (1987) 
[Mi06] Milnor, J.: Dynamics in one complex variable, 3rd ed. Annals of Mathematics Studies 160, Princeton University Press, Princeton, NJ (2006)

[Mis81] Misiurewicz, M.: On iterates of $e^{z}$. Ergod. Theor. Dyn. Syst. 1(1), 103-106 (1981)

[Ne53] Nevanlinna, R.: Eindeutige Analytische Funktionen, Zweite Auflage. Springer Verlag, Göttingen (1953)

[Qiu94] Qiu, W.: Hausdorff dimension of the $M$-set of $\lambda \exp (z)$. Acta Math. Sinica (N.S.) 10(4), 362-368 (1994)

[Re04] Rempe, L.: On a question of Herman, Baker and Rippon concerning Siegel disks. Bull. Lond. Math. Soc. 36(4), 516-518 (2004)

[Re07] Rempe, L.: On a question of Eremenko concerning escaping components of entire functions. Bull. Lond. Math. Soc. 39, 661-666 (2007)

[Re] Rempe, L.: Rigidity of escaping dynamics for transcendental entire functions. Acta Math. 203 235-267 (2009)

[RSch08] Rempe, L., Schleicher, D.: Bifurcation loci of exponential maps and quadratic polynomials: local connectivity, triviality of fibers, and density of hyperbolicity. In: M. Lyubich, M. Yampolsky (eds.) Holomorphic Dynamics and Renormalization: A Volume in Honour of John Milnor's 75th birthday. Fields Institute Communications 53, 177-196 (2008)

[RSch09] Rempe, L., Schleicher, D.: Bifurcations in the space of exponential maps. Invent. Math. 175(1), 103-135 (2009)

[Ri08] Rippon, P.: Baker domains. In: Phil Rippon, Gwyneth Stallard (eds.) Transcendental Dynamics and Complex Analysis. Lond. Math. Soc. Lecture Note Ser. 348, Cambridge University Press, Cambridge, 371-395 (2008)

[RS99a] Rippon, P.J., Stallard, G.M.: Families of Baker domains: I. Nonlinearity 12, 1005-1012 (1999a)

[RS99b] Rippon, P.J., Stallard, G.M.: Families of Baker domains II, Conform. Geom. Dyn., 3, 67-78 (1999b)

[RS00] Rippon, P.J., Stallard, G.M.: On sets where iterates of a meromorphic function zip to infinity. Bull. Lond. Math. Soc. 32, 528-536 (2000)

[RS05a] Rippon, P.J., Stallard, G.M.: On questions of Fatou and Eremenko. Proc. Am. Math. Soc. 133, 1119-1126 (2005a)

[RS05b] Rippon, P.J., Stallard, G.M.: Escaping points of meromorphic functions with a finite number of poles. J. d'Analyse Math. 96, 225-245 (2005b)

[RS] Phil J. Rippon and Gwyneth M. Stallard, Slow escaping points of meromorphic functions, Transactions of the Amer. Math. Soc., to appear, ArXiv:0812.2410v1

[Ro48] Rosenbloom, P.C.: L'itération des fonctions entières. C. R. Acad. Sci. Paris Sér. I Math. 227, 382-383 (1948)

[RoS08] Rottenfußer, G., Schleicher, D.: Escaping points of the cosine family. In: Phil Rippon, Gwyneth Stallard (eds.) Transcendental Dynamics and Complex Analysis. Lond. Math. Soc. Lecture Note Ser. 348. Cambridge University Press, Cambridge, 396-424 (2008)

[RRRS] Rottenfußer, G., Rückert, J., Rempe, L., Schleicher, D.: Dynamic rays of boundedtype entire functions. Ann. Math. to appear

[Rü08] Rückert, J.: Rational and transcendental Newton maps. In: M. Lyubich, M. Yampolsky (eds.) Holomorphic Dynamics and Renormalization: A Volume in Honour of John Milnor's 75th birthday. Fields Institute Communications 53, 197211 (2008)

[RüS07] Rückert, J., Schleicher, D.: On Newton's method for entire functions. J. Lond. Math. Soc. (2) 75, 659-676 (2007)

[S93] Schiff, J.: Normal Families. Universitext. Springer Verlag, New York (1993)

[Sch00] Schleicher, D.: Attracting dynamics for exponential maps. Ann. Acad. Sci. Fenn. 28(1), 3-34 (2003)

[Sch04a] Schleicher, D.: Hyperbolic components in exponential parameter space. Comptes Rendus - Mathématiques 339(3), 223-228 (2004) 
[Sch04b] Schleicher, D.: On fibers and local connectivity of Mandelbrot and Multibrot sets. In: M. Lapidus, M. van Frankenhuysen (eds.) Fractal Geometry and Applications: A Jubilee of Benoit Mandelbrot. Proceedings of Symposia in Pure Mathematics 72, pp. 477-507. American Mathematical Society, Providence, RI (2004)

[Sch07a] Schleicher, D.: Hausdorff dimension, its properties, and its surprises. Am. Math. Monthly 114(6), 509-528 (2007)

[Sch07b] Schleicher, D. The dynamical fine structure of iterated cosine maps and a dimension paradox. Duke Math. J. 136(2), 343-356 (2007)

[Sch08] Schleicher, D.: Newton's method as a dynamical system: efficient root finding of polynomials and the Riemann $\zeta$ function. In: M. Lyubich, M. Yampolsky (eds.) Holomorphic Dynamics and Renormalization: a Volume in Honour of John Milnor's 75th birthday. Fields Institute Communications 53, 213-224 (2008)

[SZ03a] Schleicher, D., Zimmer, J.: Escaping points for exponential maps. J. Lond. Math. Soc. (2) 67, 380-400 (2003)

[SZ03b] Schleicher, D., Zimmer, J.: Periodic points and dynamic rays of exponential maps. Ann. Acad. Sci. Fenn. 28(2), 327-354 (2003)

[Schu08] Schubert, H.: Area of Fatou sets of trigonometric functions. Proc. Am. Math. Soc. 136, 1251-1259 (2008)

[Se09] Selinger, N.: On the Boundary Behavior of Thurston's Pullback Map. In: Dierk Schleicher (ed.) Complex Dynamics: Families and Friends, pp. 585-595, Chapter 16. A K Peters, Wellesley, MA (2009)

[Sh87] Shishikura, M.: On the quasiconformal surgery of rational functions. Ann. Sci.Éc. Norm. Sup. 4e Ser. 20, 1-29 (1987)

[Sh09] Shishikura, M.: The connectivity of the Julia set and fixed points. In: Dierk Schleicher (ed.) Complex Dynamics: Families and Friends, pp. 257-276. Chapter 6. A K Peters, Wellesley, MA (2009)

[St96] Stallard, G.: The Hausdorff dimension of Julia sets of entire functions. II. Math. Proc. Cambridge Philos. Soc. 119(3), 513-536 (1996)

[St97] Stallard, G.: The Hausdorff dimension of Julia sets of entire functions. III. J. Math. Proc. Camb. Philos. Soc. 122(2), 223-244 (1997)

[St08] Stallard, G.: Dimensions of Julia sets of transcendental meromorphic functions. In: Transcendental dynamics and complex analysis, Lond. Math. Soc. Lecture Note Ser. 348, pp. 425-446. Cambridge University Press, Cambridge 2008

[Ur03] Urbański, M.: Measures and dimensions in conformal dynamics. Bull. Am. Math. Soc. (N.S.) 40(3), 281-321 (2003)

[UZ07] Urbański, M., Zdunik, A.: Instability of exponential Collet-Eckmann maps. Israel J. Math. 161, 347-371 (2007)

[Tö39] Töpfer, H.: Über die Iteration der ganzen transzendenten Funktionen, insbesondere von $\sin z$ und $\cos z$ (German), Math. Ann. 117, 65-84 (1939)

[Ye94] Ye, Z.: Structural instability of exponential functions. Trans. Am. Math. Soc. 344(1), 379-389 (1994)

[Y95] Yoccoz, J.-C.: Théorème de Siegel, nombres de Bruno et polynômes quadratiques. Petits diviseurs en dimension 1. Astérisque 231, 3-88 (1995)

[Zh06] Zheng, J.-H.: On multiply-connected Fatou components in iteration of meromorphic functions. J. Math. Anal. Appl.313(1), 24-37 (2006)

[Z75] Zalcman, L.: A heuristic principle in complex function theory. Am. Math. Monthly 82(8), 813-817 (1975) 